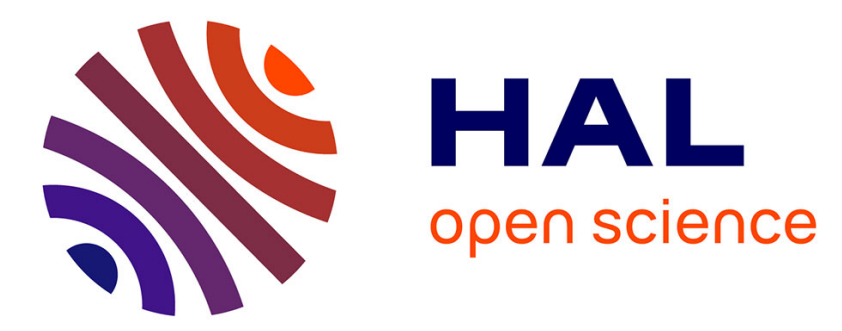

\title{
Complexity of planning for connected agents
}

Tristan Charrier, Arthur Queffelec, Ocan Sankur, François Schwarzentruber

\section{To cite this version:}

Tristan Charrier, Arthur Queffelec, Ocan Sankur, François Schwarzentruber. Complexity of planning for connected agents. Autonomous Agents and Multi-Agent Systems, 2020, 34 (2), 10.1007/s10458020-09468-5 . hal-03003144

\section{HAL Id: hal-03003144 https://hal.science/hal-03003144}

Submitted on 13 Nov 2020

HAL is a multi-disciplinary open access archive for the deposit and dissemination of scientific research documents, whether they are published or not. The documents may come from teaching and research institutions in France or abroad, or from public or private research centers.
L'archive ouverte pluridisciplinaire HAL, est destinée au dépôt et à la diffusion de documents scientifiques de niveau recherche, publiés ou non, émanant des établissements d'enseignement et de recherche français ou étrangers, des laboratoires publics ou privés. 


\title{
Complexity of Planning for Connected Agents
}

\author{
Tristan Charrier • Arthur Queffelec • \\ Ocan Sankur • François Schwarzentruber
}

Received: date / Accepted: date

\begin{abstract}
We study a variant of the Multi-Agent Path Finding (MAPF) problem in which the group of agents are required to stay connected with a supervising base station throughout the execution. In addition, we consider the problem of covering an area with the same connectivity constraint. We show that both problems are PSPACEcomplete on directed and undirected topological graphs while checking the existence of a bounded plan is NP-complete when the bound is given in unary (and PSPACEhard when the encoding is in binary). Moreover, we identify a realistic class of topological graphs on which the decision problem falls in NLOGSPACE although the bounded versions remain NP-complete for unary encoding.
\end{abstract}

Keywords Artifical Intelligence $\cdot$ Multi-Agent Systems $\cdot$ Planning $\cdot$ Computational Complexity

Mathematics Subject Classification (2010) 68T20 - 03D15

\section{Introduction}

The Multi-Agent Path Finding (MAPF) problem asks for a plan to move a group of agents to a target configuration in a graph while avoiding collisions. It is an important problem in the design of groups of autonomous vehicles, and has been used in several applications such as Kiva (Amazon Robotics) warehouse systems [58], autonomous aircraft towing vehicles [36], characters in video games [49] and office robots [57].

A closely related problem is that of coverage path planning which consists in computing a plan that visits a set of given locations in a graph. A comprehensive survey is given in [23]. Applications include underwater ship hull inspection [18], wildfire tracking with drones [40], to name a few.

Univ Rennes, Inria, CNRS, IRISA

T. Charrier · A. Queffelec · O. Sankur · F. Schwarzentruber

263 Avenue Général Leclerc, 35000 Rennes, France

E-mail: firstname.lastname@irisa.fr 
An important application area is that of information gathering missions in which agents must visit a set of locations in an area and gather information using sensors (e.g. camera, smoke sensor, hygrometer etc.). Some applications, such as search and rescue missions, might require continuous connection between all agents and a base station, for instance, in order to stream video and allow human operators to make decisions [2]. In this case, the path planning and coverage path planning algorithms must take additional connectivity constraints into account in order to compute suitable plans. Several works have explored planning algorithms in this setting e.g. [44, 39].

Some works on path planning and coverage path planning either assume a given discrete graph model obtained by cell decomposition or visibility graph [34], or applies a sampling method to construct a graph on which combinatorial algorithms are applied (see also the Related Work section below). Graph-based algorithms such as those we study, and previous work such as $A^{*}$ for multi-agent path finding $[45,52]$ and conflict-based search [48] are thus relevant in this setting. It was shown that multi-agent path planning is related to network flow problems, and that one can use algorithms for the latter to compute plans [64]. It is thus important to understand the computational complexity of these graph problems to understand the limits of the algorithmic solutions and as well as heuristics that can be applied to the problem at hand.

The variant of MAPF with connectivity constraints and related computational complexity results were studied in [26,54]. In [54], complexity results are presented for connectivity constraints with and without collision constraints and the existence of a plan of arbitrary length in both cases was shown to be PSPACE-complete in undirected graphs. Interestingly, it means that it has the same complexity as classical planning [8]. The work in [7] considers the path coverage problem and provides experiments in which instances are described in Planning Domain Description Language and solved with the planner Functional STRIPS [21]. In other words, MAPF with connectivity constraints is more difficult than MAPF with collision constraints: deciding the existence of a collision-free plan of arbitrary length is in $\mathrm{P}$, as stated in [65].

Now, concerning the optimization problems, there is a subtlety about the encoding (unary vs binary) of the length of plans. For MAPF with collision constraints, as the existence of a plan is equivalent to the existence of a plan of length $O\left(|V|^{3}\right)$ where $|V|$ is the number of nodes [65], the encoding of the length is not relevant. Thus, several papers on the topic do not specify the encoding $[5,35,41,62,63,64]$. However, for MAPF with connectivity constraints, there is no such bound on the length of a plan. We show that the existence of a bounded plan when the bound is given in binary is PSPACE-complete; while when the bound is given unary, the problem is NP-complete, even on undirected graphs, as claimed by Hollinger and Singh in [26], although they do not explicitly specify the membership proof and are ambiguous about the encoding ${ }^{1}$.

\footnotetext{
1 The bounded plan is clearly in NP when the bound $\ell$ is written in unary: simply guess a solution of length at most $\ell$ and check that it is correct. When the bound is given in binary, a solution may be in exponential size, and cannot be guessed in polynomial time.
} 
We mainly consider a setting where collisions are ignored. In fact, we are interested in the computational complexity of maintaining connectivity in plans. As in [26], agents can be assumed to be equipped with a low-level collision avoidance system in some cases. Additionally, in drone applications with few agents, different altitudes can be used to avoid collisions. Nevertheless, we do discuss the impact of taking collision constraints into account in our results as well.

In this work, we are interested in the computational complexity of two problems: Reachability is the variant of MAPF with connectivity constraints, without collision constraints; and Coverage is the variant of path coverage planning problem for multiple agents with connectivity constraints. As in [26,54], a problem instance is a topological graph, which is a set of nodes given with movement edges along which agents can move, and communication edges which determine pairs of vertices at which communication is possible. Our results are as follows. We establish the computational complexity of determining the existence of plans, showing that Reachability is PSPACE-complete on directed graphs as well, and proving that Coverage is PSPACE-complete on both types of graphs. We study bounded versions of these problems where a bound on the length of the plan is given as part of the input; these are denoted by bReachability and bCoverage. As in [56], we advocate for lengths of plans written in unary although we also study the complexity of our problems when the encoding is binary. We show that both bReachability and bCoverage are NP-complete when the bound is given in unary, and we clearly state their PSPACE-complete when the bound is given in binary.

Given the prohibitively high complexity reported above, we are interested in searching for 'easier' classes of topological graphs which are realistic for the purpose of information gathering missions. One of our main contributions is the identification of a natural class of topological graphs, called sight-moveable, for which we give efficient algorithms. This class requires that whenever an agent can communicate with another node, then it can also move to that node while maintaining direct communication. This can be seen as a restriction on allowed topological graphs; however, if the graph at hand does not have this property, it may be possible to enforce it by removing some communication edges (not allowing the planning algorithm to rely on those edges). Subsection 6.3 describes a way to obtain sight-moveable graphs from a given topological graph. Thus, any plan found in the obtained sight-moveable graph can be applied on the original one.

The class of sight-moveable graphs offers good computational properties: both Reachability and Coverage belong to NLOGSPACE, meaning that they can be solved by a non-deterministic algorithm that only uses a logarithmic amount of memory. Practically, it means that algorithms for generating plans can be parallelized, because NLOGSPACE is included in the Nick's class (NC), [16], known to represent decision problems for which there is a parallel algorithm. However, the bounded versions remain NP-complete. We complete the investigation with the complexity analysis of the problem with complete communication graphs, where all pairs of nodes can communicate. In addition, we give complexity results on several variants of our problems.

Note that the PSPACE lower bound given in [54] concerned the reachability problem where agents start from arbitrary locations, whereas we prove that the PSPACE 
lower bound still holds for agents starting all from the base. Furthermore, this article extends the work of $[11,12]$, in which the complexity of Reachability (when agents start from the base) and Coverage on undirected topological graphs was left open. In this article, we solved them (Theorem 3 and 4). We also include detailed proofs and discuss relevant extensions that were not considered in previous work.

Overview We first discuss related work in Section 2. In Section 3, we introduce the required notions for the rest of the paper. In Section 4, we describe the upper bounds of our problems on directed topological graphs and, in Section 5, we prove the lower bounds on undirected topological graphs to obtain completeness results. In Section 6, we study sight-moveable topological graphs. Section 7 contains the complexity analysis for complete-communication topological graphs. We introduce relevant extensions of our problems, in Section 8. We present a conclusion and future works in Section 9.

\section{Related Work}

Planning problems for multiple agents were considered in the robot motion planning setting, for instance the case of two disc-shaped robots moving in the presence of polygonal obstacles [47]. The planning problem for multiple agents is PSPACE-hard even when robot shapes are restricted to simple tiles [25]. PSPACE-hardness was shown in case of unit-square robots with polygonal obstacles as well when agents are unlabelled/homogeneous, or in our terminology, anonymous [51]. A line of algorithms that avoid this high complexity are those based on sampling methods such as $[29,32]$. These randomly sample points and check whether these points can be connected respecting the constraints of the system. This allows one to quickly generate a tree or a graph between sampled points, and continue sampling points until the desired plan is found.

The coverage path planning problem has been studied in different settings; see the surveys $[14,23,9]$. This problem can be used to solve inspection (where a given set of points of interests must be observed), surveillance (points of interest must be continuously visited) problems, and can be used in applications such as lawn mowing and floor cleaning. Sampling-based algorithm were given in this setting as well. One can prove probabilistic completeness results in some cases, which means that if a solution exists, then the algorithm will eventually find it with probability 1; although it may not be able to detect that no solution exists [18]. Some of these algorithms work in two phases: the first phase consists in sampling points and checking the feasibility of the edges between them, and the second phase consists in solving a graph problem on this structure, and repeating phase 1 if no solution is found. Some works use approximations of metric traveling salesman problem (TSP) to find solutions in the constructed graph $[17,19]$. Other works use $A^{*}$ search algorithms with suitable heuristics to compute approximate solutions [22].

Several works consider the coverage path planning problem for multiple agents [13, 23,9]. In [33], a centralized framework is given to compute plans for the persistent coverage problem without communication constraints. In [43], algorithms are given 
for the repeated coverage problem in unknown environments for a team of robots, including the case of the line-of-sight communication restriction. This uses the cell decomposition given in [15] for the coverage path planning. In [24,59] a graph structure is obtained thanks to a cell decomposition, and variants of spanning trees are computed on this graph to ensure coverage with multiple robots. Graph problems related to this problem are studied in [60]. The generalization of traveling salesman problem was studied in [3] which is a relevant problem for coverage path planning in the multi-agent setting. Some works also consider maneuverability and camera angle constraints [1].

The continuous communication restriction appears in several works, e.g. $[39,37$, $61,55]$ which provide experiments to demonstrate the feasibility of the proposed algorithms. Some works consider the use of dynamic teams of robots which exchange their information on current solutions [38]. Communication and battery restrictions are considered together in [10] which gives an algorithm in which robots can adopt the roles for exploring, meeting, sacrificing themselves (continuing the mission in case of low battery), and serving as communication relay for other robots. Different communication restrictions have been considered as well [2] such as event-based communication where the discovery of new information triggers communication (see e.g [6]).

\section{Preliminaries}

In some path finding applications, one considers a discretization of the space which yields a graph of movements on which algorithms are run. For instance, regular grids which decompose the space in square, triangular or hexagonal cells, irregular grids with techniques such as quadtrees [20,31] or Voronoï diagrams comprehensively discussed in the survey [4].

Our work is independent of the particular method used to obtain the discretization. We only work under the hypothesis that a feasible plan on the graph generated by the discretization is also feasible in the continuous space.

We will, now, introduce the notions used throughout this work. We define formally the general topological graphs and some subclasses, the notion of execution and the properties considered, the decision problems we investigate and some known results on these problems.

\subsection{Topological Graphs}

Our problems require graphs with two types of edges: movement edges along which agents can move, and communication edges which specify whether agents at two different locations can communicate. We call graphs with this additional information topological graphs. The formal definition is the following and examples are depicted in Figure 1.

Definition 1 (Topological Graph) A topological graph is a tuple $G=\left\langle V, E_{m}, E_{c}\right\rangle$, with $V$ a finite set of nodes containing a distinguished node $B$ called the base, $E_{m} \subseteq$ 


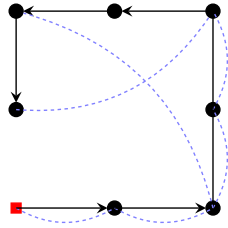

(a) Directed

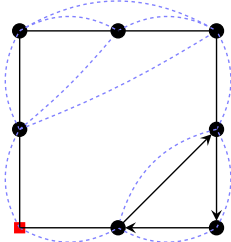

(c) Sight-moveable

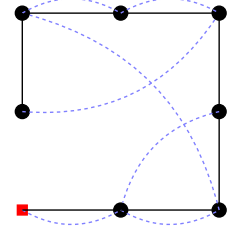

(b) Undirected

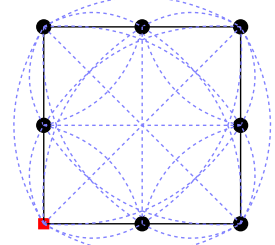

(d) Complete-communication

$\begin{array}{lll}\text { - Base } & \bullet & \text { Node } \\ \text { - Movement } & \cdots & \text { Communication }\end{array}$

Fig. 1: Examples of topological graphs.

$V \times V$ a set of movement edges and $E_{c} \subseteq V \times V$ a set of undirected communication edges.

The node $B$ is the supervision base station from which the agents start the mission and with which they are required to keep communication.

In some applications movement edges are reversible, that is, if an agent can travel from a node to another, it can also go back to the former through the same edge. Undirected graphs thus naturally arise in some applications. See Figure $1 \mathrm{~b}$ for an example.

Definition 2 (Undirected Topological Graph) A topological graph is said to be undirected if $\left\langle V, E_{m}\right\rangle$ is an undirected graph.

Let us now introduce our new class, called sight-moveable topological graphs, which is one of our main contributions. This class requires the movement edges to be reflexive. In addition, whenever an agent can communicate with another node, then it can also move to that node while maintaining the communication without using another agent as a relay. An example of a sight-moveable graph is given in Figure 1c.

Definition 3 (Sight-Moveable Topological Graph) A sight-moveable topological graph $G=\left\langle V, E_{m}, E_{c}\right\rangle$ is a directed topological graph such that

1. $E_{m} \subseteq E_{c}$,

2. for all $v \in V,(v, v) \in E_{m}$,

3. and whenever $\left(v, v^{\prime}\right) \in E_{c}$, there exists a sequence $\rho=\left\langle\rho_{1}, \ldots, \rho_{n}\right\rangle$ of nodes such that $v=\rho_{1}, v^{\prime}=\rho_{n},\left(v, \rho_{i}\right) \in E_{c}$ and $\left(\rho_{i}, \rho_{i+1}\right) \in E_{m}$ for all $i \in$ $\{1, \ldots, n-1\}$. 
Last, we define the complete-communication topological graphs which are simply sight-moveable topological graphs with undirected movement and complete communication topology. An example of such a graph is depicted in Figure 1d, and the formal definition is the following.

Definition 4 (Complete-Communication Topological Graph) A topological graph is said to be a complete-communication if it is a sight-moveable topological graph such that $\left\langle V, E_{m}\right\rangle$ is an undirected graph and $E_{c}=V \times V$.

Observe that a complete-communication graphs are reflexive, undirected, connected graphs with communication edges between each pair of nodes.

We say that a topological graph has a planar (grid) movement graph iff the graph $\left\langle V, E_{m}\right\rangle$ is a planar (resp. grid) graph.

\subsection{Execution}

An execution is a finite sequence of configurations describing the positions of the agents during the mission. We require that all agents should be connected to the base in all configurations. We will use multi-sets to denote nodes occupied by agents in configurations, since agents are anonymous. In other terms, if the goal is to reach a target configuration, it does not matter which agent occupies which node, as long as there is the right number of agents at each node. Other works use the term unlabelled or homogeneous (see e.g. [51]); but we use the terminology of MAPF.

The formal definition of a configuration is the following.

Definition 5 (Configuration) A configuration $c$ of $n$ agents in a topological graph $G$ is a multi-set of elements of $V$ of size $n$, denoted $c=\left\langle c_{1}, \ldots, c_{n}\right\rangle$.

A configuration is said to be connected iff the graph $\left\langle V_{c}, E_{c} \cap\left(V_{c} \times V_{c}\right)\right\rangle$ is connected with $V_{c}=\left\{B, c_{1}, \ldots, c_{n}\right\}$.

Given a topological graph $G=\left\langle V, E_{m}, E_{c}\right\rangle$, we write $c \rightarrow_{G} c^{\prime}$ to say that agents in $c$ perform one-step movements to occupy nodes in $c^{\prime}$. Formally, we have $c \rightarrow_{G} c^{\prime}$ if $c$ (resp. $\left.c^{\prime}\right)$ can be written as $\left\langle c_{1}, \ldots, c_{n}\right\rangle$ (resp. $\left.\left\langle c_{1}^{\prime}, \ldots, c_{n}^{\prime}\right\rangle\right)$, and $\left(c_{i}, c_{i}^{\prime}\right) \in E_{m}$ for all $1 \leq i \leq n$.

Definition 6 (Execution) An execution $e$ of length $\ell$ with $n$ agents in a topological graph $G$ is a sequence of connected configuration $\left\langle c^{1}, \ldots, c^{\ell}\right\rangle$ such that $c^{j} \rightarrow_{G} c^{j+1}$ for all $1 \leq i<\ell$.

In our setting, the makespan of an execution is equal to its length.

A covering execution $e=\left\langle c^{1}, \ldots c^{\ell}\right\rangle$ of length $\ell$ with $n$ agents in a graph $G$ is an execution such that $c^{1}=c^{\ell}=\langle B, \ldots, B\rangle$ and for all $v \in V$, there exists $j \in\{1, \ldots, \ell\}$ such that $v$ appears in $c^{j}$. An example of such an execution is depicted in Figure 2 (from left to right). 


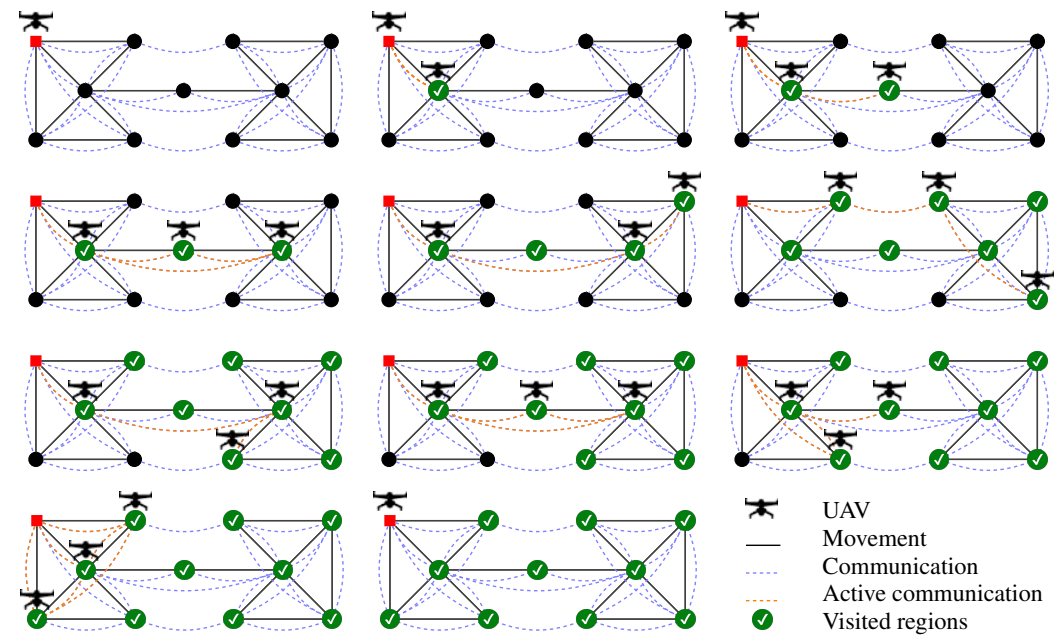

Fig. 2: Example of a covering execution of length 10 with 3 agents. The plan is depicted from left to right and from top to bottom.

\subsection{Decision Problems}

We formally define the problems Reachability and Coverage and their bounded versions bReachability and bCoverage.

\subsubsection{The Unbounded Case}

Definition 7 (Reachability) Given a topological graph $G$, an integer $n$ and a configuration $c$ of size $n$, decide if there is an execution $\left\langle c^{1}, \ldots, c^{\ell}\right\rangle$ in $G$ such that $c^{1}=\langle B, \ldots, B\rangle$ and $c^{\ell}=c$.

Definition 8 (Coverage) Given a topological graph $G$ and an integer $n$, decide if there exists a covering execution with $n$ agents.

We also consider variants Reachability-init and Coverage-init denote the problems in which the agents start at a given configuration rather than at the base. In other words, the initial configuration is part of the input. In addition, Coverage-init requires the agents to return to the initial configuration rather than to the base.

In the above problems, the encoding of the integer $n$ (unary or binary) does not matter. Indeed, in Reachability, Reachability-init and Coverage-init, the input already contains some configuration which is of size $n$; in Coverage, it is useless to have $n$ greater than the number of nodes.

\subsubsection{The Bounded Case}

The bounded versions are inspired from the so-called polynomial-length planning problem [56] in which we ask for the existence of a plan of length bounded by a 
polynomial in the size of the planning task. This can be seen as the decision problem for the optimization problem that seeks to minimize the length of an execution, except that we assume that the bound is given in unary. In fact, the goal of planning algorithms is to compute plans, so given a bound $\ell$ on the length of the desired plan, the algorithm always allocates memory space of size $\Omega(\ell)$ to store the plan.

That is why we use unary encoding in the following definitions. Binary encoding of the length $\ell$ is discussed as well in Subsection 8.1.

Definition 9 (bReachability) Given a topological graph $G$, an integer $n$ and a configuration $c$ of size $n$ and $\ell$ an integer written in unary, decide if there is an execution $\left\langle c^{1}, \ldots, c^{\ell^{\prime}}\right\rangle$ in $G$ s.t. $\ell^{\prime} \leq \ell$ and $c^{\ell^{\prime}}=c$.

Definition 10 (bCoverage) Given a topological graph $G$, an integer $n$, an integer $\ell$ written in unary, decide if there exists a covering execution of length $\ell^{\prime}$ such $\ell^{\prime} \leq \ell$.

\subsubsection{Restriction to Subclasses of Graphs}

We consider the restriction of the above problems to the following subclasses of graphs: directed graphs (denoted by dir), undirected graphs (denoted by und), sightmoveable graphs (denoted by $\mathrm{sm}$ ) and complete-communication graphs (denoted by cc). The variants of these problems to a given graph class will be denoted using a subscript, that is, Reachability ${ }_{\star}$, Coverage $\mathbf{C}_{\star}$, bReachability $_{\star}$, bCoverage $\mathbf{b}_{\star}$, Reachability-init ${ }_{\star}$, and Coverage-init ${ }_{\star}$ denote the restriction of these problems to graphs of type $\star \in\{$ und, dir, sm, cc $\}$.

\subsection{Known Results}

The connected version of MAPF was introduced in [26], in which a topological graph discretizes the space and it is proved that the existence of a plan for the reachability of a configuration of non-anonymous agents in a bounded number of steps with collisions allowed is NP-hard:

\section{Theorem 1 ([26]) bReachability-init ${ }_{\text {und }}$ is NP-hard.}

As stated before, the above paper actually states the NP-completeness of this problem but without specifying the encoding of the bound.

Tateo et al., in [54], establish the complexity of Reachability-init ${ }_{\text {und }}$ :

\section{Theorem 2 ([54]) Reachability-init ${ }_{\mathrm{und}}$ is PSPACE-complete.}

The setting of Tateo et al. is identical to ours with the exception that the starting configuration is part of the input in their case. We relate both problems showing PSPACE-hardness when agents all start at the base. 


\begin{tabular}{|c|c|c|c|c|}
\hline Top. Graph/Problem & $\begin{array}{l}\text { Reachability } \\
\text { (Def. 7) }\end{array}$ & $\begin{array}{c}\text { Coverage } \\
\text { (Def. 8) }\end{array}$ & $\begin{array}{l}\text { bReachability } \\
\text { (Def. 9) }\end{array}$ & $\begin{array}{l}\text { bCoverage } \\
\text { (Def. 10) }\end{array}$ \\
\hline $\begin{array}{l}\text { Directed } \\
\text { (Def. 1) }\end{array}$ & $\begin{array}{l}\text { PSPACE-complete } \\
\text { (Th. 3) }\end{array}$ & \multirow{2}{*}{$\begin{array}{c}\text { PSPACE-complete } \\
\text { (Th. 4) }\end{array}$} & \multirow{2}{*}{$\begin{array}{c}\text { NP-complete } \\
{[26]}\end{array}$} & \multirow{4}{*}{$\begin{array}{l}\text { NP-complete } \\
\text { (Th. 8) }\end{array}$} \\
\hline $\begin{array}{l}\text { Undirected } \\
\text { (Def. 2) }\end{array}$ & $\begin{array}{l}\text { PSPACE-complete } \\
{[54]}\end{array}$ & & & \\
\hline $\begin{array}{l}\text { Sight-Moveable } \\
\text { (Def. 3) }\end{array}$ & \multirow{2}{*}{$\begin{array}{l}\text { in LOGSPACE } \\
\text { (Prop. 3) }\end{array}$} & \multirow{2}{*}{$\begin{array}{l}\text { in NLOGSPACE } \\
\text { (Prop. 4) }\end{array}$} & $\begin{array}{l}\text { NP-complete } \\
\text { (Th. 6) }\end{array}$ & \\
\hline $\begin{array}{l}\text { Complete-Comm. } \\
\text { (Def. 4) }\end{array}$ & & & $\begin{array}{l}\text { in NLOGSPACE } \\
\text { (Prop. 5) }\end{array}$ & \\
\hline
\end{tabular}

Fig. 3: Overview of the complexity results.

\subsection{Overview of Results}

In the rest of the paper, we study upper and lower complexity bounds for the defined decision problems on different topological graphs. The following sections present our results, respectively, for the general case, the undirected graphs, sight-moveable graphs, and complete-communication graphs. An overview of these results is given in Figure 3.

\section{Directed Topological Graphs}

In this section, we will consider the previous problems restricted to the class of directed topological graphs. These problems are thus denoted by Reachability $\mathbf{d i r}_{\mathrm{dir}}$ and bReachability $_{\mathrm{dir}}\left(\right.$ resp. Coverage $\mathrm{dir}_{\mathrm{dir}}$ and bCoverage $_{\mathrm{dir}}$ ), with dir denoting the class of directed topological graphs.

In this section, we show upper bounds for all our problems in the general case, that is, for directed topological graphs. Observe that this also provides upper bounds for other classes such as undirected graphs.

For the unbounded problems, we can design a straightforward non-deterministic algorithm running in polynomial space, that guesses an execution by keeping in memory the last configuration, and, for Coverage, the set of visited regions. In fact, the number of configurations is exponential, and a single configuration can be stored in polynomial space. Moreover, one can easily bound the length of executions by an exponential as well. We conclude with Savitch's Theorem (NPSPACE=PSPACE)[46]:

\section{Proposition 1 Coverage and Reachability are in PSPACE.}

For the bounded versions of the problems, since the bound is encoded in unary, one can guess and check a path of bounded length in polynomial time. The result follows.

\section{Proposition 2 bCoverage and bReachability are in NP.}

\section{Undirected Topological Graphs}

In this section we prove the PSPACE lower bound of the problems Reachability and Coverage on undirected topological graphs. 


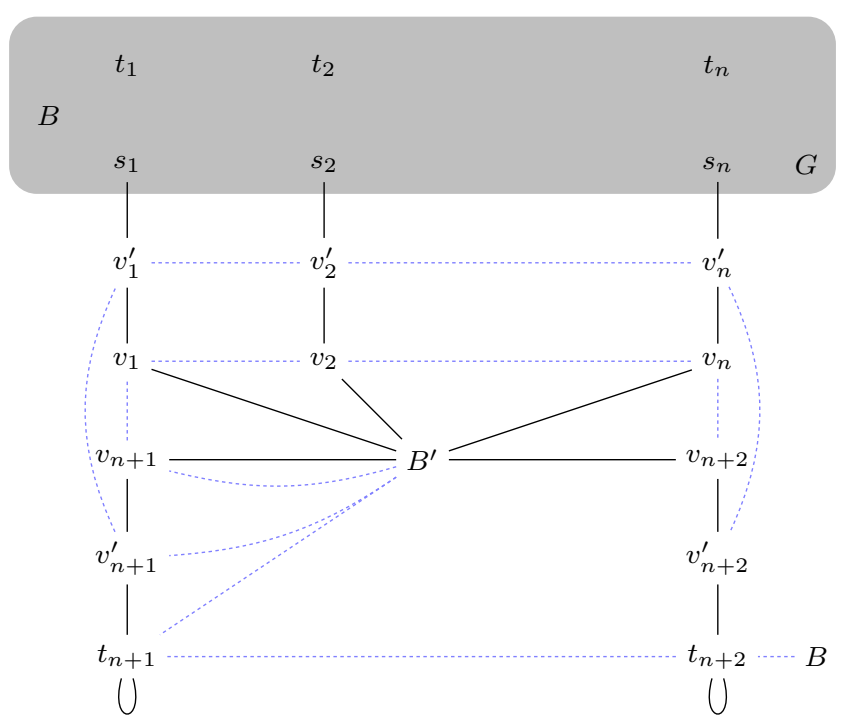

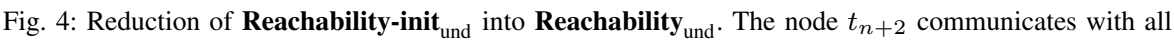
nodes $v$ such that $v$ communicates with $B$.

We consider the result of Theorem 2 in the setting where all agents start at the base.

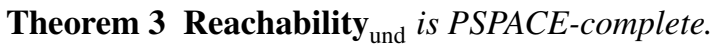

Proof The upper bound comes from Proposition 1. The lower bound is by reduction from Reachability-init ${ }_{\text {und }}$ (see Theorem 2). Let us denote by a tuple $(G, B, n, s, t)$ the instances of Reachability-init ${ }_{\text {und }}$ where $G$ is the graph, $B$ the base, $n$ the number of agents, $s$ the initial configuration and $t$ the target configuration. Instances of Reachability $_{\text {und }}$ will be denoted by $(G, B, n, t)$ as the initial configuration is fixed.

Let $(G, B, n, s, t)$ be an instance of Reachability-init ${ }_{\text {und }}$. We show how to map

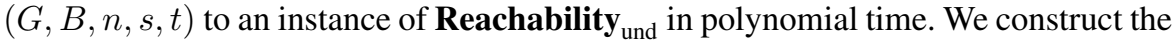
instance $\left(G^{\prime}, B^{\prime}, n+2, t^{\prime}\right)$ of Reachability ${ }_{\text {und }}$ where $G^{\prime}$ is given in Figure $4, B^{\prime}$ is the base, and the final configuration $t^{\prime}$ is $\left\langle t_{1}, \ldots, t_{n}, t_{n+1}, t_{n+2}\right\rangle$.

Let us describe more precisely the construction of $G^{\prime}$. We write $s=\left\langle s_{1}, \ldots, s_{n}\right\rangle$ and $t=\left\langle t_{1}, \ldots, t_{n}\right\rangle$. The graph $G^{\prime}$ contains the graph $G$ : in particular, it contains $B$ that is no longer the base; a new node $B^{\prime}$ is now the base. Let us describe the construction of $G^{\prime}$.

Nodes We first create two layers of $n+2$ vertices. The first layer is composed of $v_{1}, \ldots, v_{n}, v_{n+1}, v_{n+2}$ and the second of $v_{1}^{\prime}, \ldots, v_{n}^{\prime}, v_{n+1}^{\prime}, v_{n+2}^{\prime}$. We also add two nodes $t_{n+1}$ and $t_{n+2}$.

Movement We add movement edges between $t_{n+1}$ and $v_{n+1}^{\prime}, t_{n+2}$ and $v_{n+2}^{\prime}, t_{n+1}$ and $t_{n+1}$, and $t_{n+2}$ and $t_{n+2}$. Then, the role of $t_{n+1}$ and $t_{n+2}$ is to relay the communication from $B^{\prime}$ to nodes in $G$. We connect $B^{\prime}$ to the first layer, i.e. with 
a movement edge between $B^{\prime}$ and $v_{i}$, for all $1 \leq i \leq n+2$. The first layer has movement edges to the second layer, i.e. with a movement edge between $v_{i}$ and $v_{i}^{\prime}$, for all $1 \leq i \leq n+2$. The $n$ first vertices of the second layer has movement edges to the initial configuration $s$ such that there is a movement edge from $v_{i}^{\prime}$ to $s_{i}$, for all $1 \leq i \leq n$.

Communication We add communication edges from $B^{\prime}$ to $t_{n+1}$, from $t_{n+1}$ to $t_{n+2}$ as well as from $t_{n+2}$ to $B$ and if there exists $v$ such that $B$ communicates $v$ then we create a communication edge from $t_{n+2}$ to $v$. We add a communication edge from $B^{\prime}$ to $v_{n+1}$, from $v_{i}$ to $v_{i+1}$, for all $1 \leq i<n$, from $v_{n+1}$ to $v_{1}$ and from $v_{n}$ to $v_{n+2}$. We repeat this last procedure for the second layer as well.

We now give the formal definition of our reduction. In the sequel, we use the symbol $\sqcup$ to emphasize that the union is of disjoint sets. Formally, given $(G, B, n, s, t)$ with $G=\left\langle V, E_{m}, E_{c}\right\rangle$ with base $B$, we define $\left(G^{\prime}, B^{\prime}, n+2, t^{\prime}\right)$ where $G^{\prime}=$ $\left\langle V^{\prime}, E_{m}^{\prime}, E_{c}^{\prime}\right\rangle$ with base $B^{\prime}$ where:

$-V^{\prime}:=V \sqcup\left\{B^{\prime}, v_{1}, \ldots, v_{n}, v_{1}^{\prime}, \ldots, v_{n}^{\prime}, v_{n+1}, v_{n+2}, v_{n+1}^{\prime}, v_{n+2}^{\prime}, t_{n+1}, t_{n+2}\right\}$

- $E_{m}^{\prime}$ is the symmetric closure of

$$
\begin{aligned}
& E_{m} \cup\left\{\left(B^{\prime}, v_{1}\right), \ldots,\left(B^{\prime}, v_{n}\right),\left(B^{\prime}, v_{n+1}\right),\left(B^{\prime}, v_{n+2}\right)\right\} \\
& \cup\left\{\left(v_{1}, v_{1}^{\prime}\right), \ldots,\left(v_{n}, v_{n}^{\prime}\right)\right\} \cup\left\{\left(v_{1}^{\prime}, s_{1}\right), \ldots,\left(v_{n}^{\prime}, s_{n}\right)\right\} \\
& \cup\left\{\left(v_{n+1}, v_{n+1}^{\prime}\right),\left(v_{n+2}, v_{n+2}^{\prime}\right),\left(v_{n+1}^{\prime}, t_{n+1}\right),\left(v_{n+2}^{\prime}, t_{n+2}\right)\right\} \\
& \cup\left\{\left(t_{n+1}, t_{n+1}\right),\left(t_{n+2}, t_{n+2}\right)\right\} ;
\end{aligned}
$$

- $E_{c}^{\prime}$ is the symmetric closure of

$$
\begin{aligned}
& E_{c} \cup\left\{\left(v_{1}^{\prime}, v_{2}^{\prime}\right), \ldots,\left(v_{n-1}^{\prime}, v_{n}^{\prime}\right)\right\} \cup\left\{\left(v_{1}, v_{2}\right), \ldots,\left(v_{n-1}, v_{n}\right)\right\} \\
& \cup\left\{\left(B^{\prime}, v_{n+1}\right),\left(B^{\prime}, v_{n+1}^{\prime}\right),\left(B^{\prime}, t_{n+1}\right)\right\} \\
& \cup\left\{\left(v_{1}^{\prime}, v_{n+1}^{\prime}\right),\left(v_{n}^{\prime}, v_{n+2}^{\prime}\right),\left(t_{n+2}, B\right)\right\} \\
& \cup\left\{\left(t_{n+2}, v\right) \mid(B, v) \in E_{c}\right\} .
\end{aligned}
$$

It is worth noting that all connected configurations in $G$ are now connected via $B^{\prime}$, $t_{n+1}$ and $t_{n+2}$. We now show the instance $(G, B, n, s, t)$ of Reachability-init und $_{\text {is }}$ feasible if, and only if in the constructed instance $\left(G^{\prime}, B^{\prime}, n+2, t^{\prime}\right)$ of Reachability is feasible.

$(\Rightarrow)$ Suppose that $t$ is reachable from $s$ in the instance $(G, B, n, s, t)$. We construct an execution for $\left(G^{\prime}, B^{\prime}, n+2, t^{\prime}\right)$ as follows. All agents start from $B^{\prime}$. The $n+2$ agents first reach $\left\langle v_{1}, \ldots, v_{n+2}\right\rangle$, then $\left\langle v_{1}^{\prime}, \ldots, v_{n+2}^{\prime}\right\rangle$, then $\left\langle s_{1}, \ldots, s_{n}, t_{n+1}, t_{n+2}\right\rangle$. After that, the agents at positions $s_{1}, \ldots, s_{n}$ reach positions $t_{1}, \ldots, t_{n}$ (following the same plan as in for $(G, B, s, t)$ ), while the two others remain in $t_{n+1}$ and $t_{n+2}$.

$(\Leftarrow)$ Let us consider an execution $e$ from $\left\langle B^{\prime}, \ldots, B^{\prime}\right\rangle$ to $t^{\prime}$ for $\left(G^{\prime}, B^{\prime}, n+2, t^{\prime}\right)$. Let us extract an execution from $s$ to $t$ for $(G, B, n, s, t)$. In order to do so, we prove the Facts 1 and 2.

Fact 1 The configuration $\left\langle s_{1}, \ldots, s_{n}, t_{n+1}, t_{n+2}\right\rangle$, up to a permutation, appears in the execution $e$.

Proof The configuration $t^{\prime}$ is reached at the end of the execution $e$ and the $n+2$ agents started at $B^{\prime}$. Thus, $n$ agents must enter into $G$ and be at one of the $s_{i}$ at some point. Let us consider the moment of the execution $e$ when an agent, denoted $a$, occupies a node $s_{i}$. For that, it must be at $v_{i}^{\prime}$ before going to $s_{i}$. Furthermore, for agent $a$ to 
be connected at $v_{i}^{\prime}$ the nodes $v_{n+1}^{\prime}$ and $v_{1}^{\prime}, \ldots, v_{i-1}^{\prime}$ must be occupied. At the next step, at least the nodes $t_{n+1}$ and $t_{n+2}$ must be occupied for agent $a$ to be connected at $s_{i}$. Hence, when $a$ is at $v_{i}^{\prime}$, the agent which will occupy $t_{n+2}$ must be at $v_{n+2}^{\prime}$, thus, forcing $v_{i+1}^{\prime}, \ldots, v_{n}^{\prime}$ to be occupied. No agent in this configuration can move to a node $v_{i}$ of the first layer. Indeed, this agent would be disconnected, lacking a relay at $v_{n+1}$. Therefore, the next configuration must be $\left\langle s_{1}, \ldots, s_{n}, t_{n+1}, t_{n+2}\right\rangle$.

Now, by Fact 1, we can consider the last time at which the agents are in the configuration $\left\langle s_{1}, \ldots, s_{n}, t_{n+1}, t_{n+2}\right\rangle$. We prove the Fact 2 .

Fact 2 Between that last time and the end of the execution, there are always the same $n$ agents in $G$, one agent on $t_{n+1}$ and one on $t_{n+2}$.

Proof A similar reasoning to the last one can be used to show that if an agent is located at $s_{i}$ before moving to $v_{i}^{\prime}$ then nodes from $s_{1}$ to $s_{n}$ as well as $t_{n+1}$ and $t_{n+2}$ are occupied. Thus, since this is the last time the agents are in the configuration $\left\langle s_{1}, \ldots, s_{n}, t_{n+1}, t_{n+2}\right\rangle$, none of the $n$ first agents can move out of $G$. Furthermore, $t_{n+1}$ and $t_{n+2}$ must stay occupied in order for the $n$ agents in $G$ to be connected to $B^{\prime}$.

From Facts 1 and 2, the positions of the first $n$ agents in the portion of the execution between the last time in $\left\langle s_{1}, \ldots, s_{n}, t_{n+1}, t_{n+2}\right\rangle$ and $t^{\prime}$ are fully in $G$ and gives an execution starting at $s$ and finishing at $t$ for $(G, B, n, s, t)$.

We now turn our attention to Coverage $_{\text {und }}$. We start by establishing the PSPACEcompleteness of Coverage-init $_{\text {und }}$, and then show how to reduce this problem to Coverage $_{\text {und }}$.

Lemma 1 Coverage-init ${ }_{\text {und }}$ is PSPACE-complete.

Proof The membership of Coverage-init ${ }_{\text {und }}$ to PSPACE can be shown by using the same arguments as for the proof of Proposition 1. The proof of PSPACE-hardness is obtained by polynomial reduction from Reachability und $_{\text {. We map a }}$ Reachability $_{\text {und }}$-instance $(G, B, n, t)$ to the Coverage-init und $_{\text {-instance }}\left(G^{\prime}, B, 2 n, s\right)$ where $G^{\prime}$ is depicted in Figure 5 and starting at the configuration $s=\left\langle B, \ldots, B, v_{1}^{1}, \ldots, v_{n}^{1}\right\rangle$. The definition of $s$ means that $n$ agents start at the base $B$ and $n$ agents start in positions $v_{1}^{1}, \ldots, v_{n}^{1}$, that are in the reduction gadget. Let us describe the construction of $G^{\prime}$.

Nodes We make four layers of vertices from $v_{1}^{1}, \ldots, v_{n}^{1}$ to $v_{1}^{4}, \ldots, v_{n}^{4}$.

Movement We create movement edges between $v_{i}^{j}$ and $v_{i}^{j+1}$, with $1 \leq i \leq n$ and $1 \leq j \leq 3$. Each node of the first and last layers have self-loops. The node $v_{1}^{4}$ has a movement edge to all nodes of $G$.

Communication The node $v_{n}^{4}$ has a communication edge to all nodes of $G$ and all nodes of the fourth layer. We create a communication edge between $v_{i}^{2}$ to $t_{i}$, for all $1 \leq i \leq n$. We create a communication edge between $v_{i}^{3}$ and $v_{i+1}^{3}$, for all $1 \leq i<n$. Finally, we connect in communication the nodes of the first layer and the node $v_{1}^{3}$ to the base $B$. 


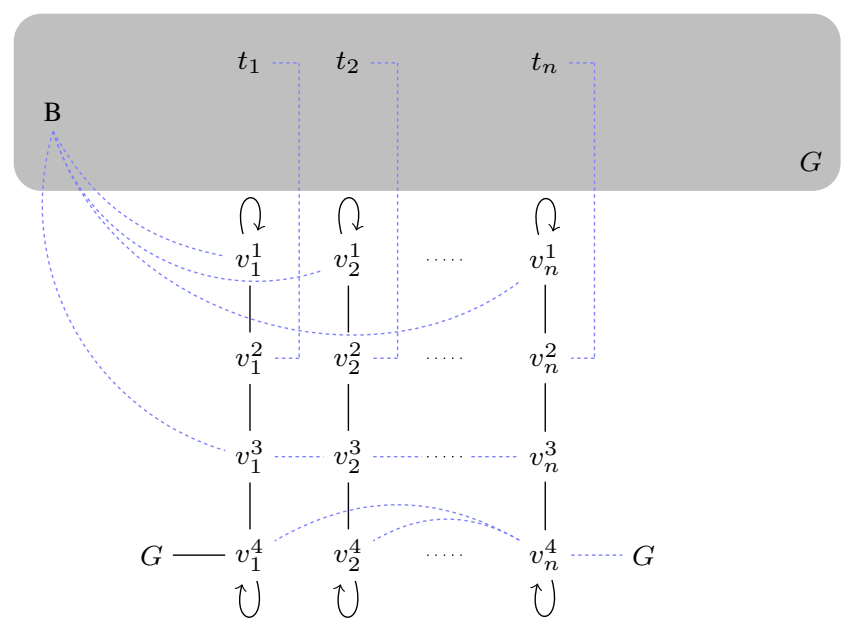

Fig. 5: Reduction of Reachability und $_{\text {to }}$ Coverage-init und $_{\text {. }}$

Formally, given $(G, B, n, t)$ with $G=\left\langle V, E_{m}, E_{c}\right\rangle$ with base $B$, we define $\left(G^{\prime}, B, 2 n, s\right)$ where $G^{\prime}=\left\langle V^{\prime}, E_{m}^{\prime}, E_{c}^{\prime}\right\rangle$ with base $B$ where:

$-V^{\prime}:=V \sqcup\left\{v_{1}^{1}, \ldots, v_{n}^{1}, v_{1}^{2}, \ldots, v_{n}^{2}, v_{1}^{3}, \ldots, v_{n}^{3}, v_{1}^{4}, \ldots v_{n}^{4}\right\}$

$-E_{m}^{\prime}$ is the symmetric closure of

$$
\begin{aligned}
& \cup E_{m} \cup\left\{\left(v, v_{1}^{4}\right) \mid v \in V\right\} \\
& \cup\left\{\left(v_{i}^{1}, v_{i}^{1}\right),\left(v_{i}^{1}, v_{i}^{2}\right),\left(v_{i}^{2}, v_{i}^{3}\right),\left(v_{i}^{3}, v_{i}^{4}\right),\left(v_{i}^{4}, v_{i}^{4}\right) \mid i \in\{1, \ldots, n\}\right\}
\end{aligned}
$$

$-E_{c}^{\prime}$ is the symmetric closure of

$$
\begin{aligned}
& E_{c} \cup\left\{\left(B, v_{1}^{1}\right) \ldots\left(B, v_{n}^{1}\right)\right\} \cup\left\{\left(t_{1}, v_{1}^{2}\right), \ldots,\left(t_{n}, v_{n}^{2}\right)\right\} \\
& \cup\left\{\left(B, v_{1}^{3}\right),\left(v_{1}^{3}, v_{2}^{3}\right), \ldots,\left(v_{n-1}^{3}, v_{n}^{3}\right)\right\} \\
& \cup\left\{\left(v_{1}^{4}, v_{n}^{4}\right) \ldots\left(v_{n-1}^{4}, v_{n}^{4}\right)\right\} \cup\left\{\left(v_{n}^{4}, v\right) \mid v \in V\right\} ;
\end{aligned}
$$

and $s=\left\langle B, \ldots, B, v_{1}^{1}, \ldots, v_{n}^{1}\right\rangle$.

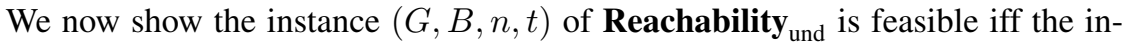
stance $\left(G^{\prime}, B, 2 n, s\right)$ of Coverage-init ${ }_{\text {und }}$ is feasible.

$(\Rightarrow)$ Suppose the configuration $t$ is reachable in $G$. Let us construct an execution starting at $s$ in $G^{\prime}$ that cover all nodes in $G^{\prime}$. First, the first $n$ agents reach configuration $t$, while the other $n$ agents stay at layer $v_{i}^{1}$ using the self-loops. After that, the first $n$ agents stay at $t$ while the others progress to the fourth layer $v_{i}^{4}$. Finally, while the agents at $v_{2}^{4}$ to $v_{n}^{4}$ don't move using the self loops, the agent at $v_{1}^{4}$ covers the whole graph since the presence of an agent at $v_{n}^{4}$ makes sure that all nodes of $G$ are connected to other agents and to the base. Once finished, this agent can go back to $v_{1}^{4}$ and the $n$ agents can return back to the layer 1 as initially. Finally, the $n$ agents occupying configuration $t$ can go back to $B$ by following the same path in reverse. This constitutes a covering execution for Coverage-init ${ }_{\text {und }}$.

$(\Leftarrow)$ Assume that $G^{\prime}$ can be covered, and let $T$ denote the first time $v_{n}^{4}$ is visited. The agent at $v_{n}^{4}$ can only be at $v_{n}^{3}$ at time $T-1$. Thus, the nodes $v_{1}^{3}, \ldots, v_{n-1}^{3}$ must also be occupied due to connectivity constraint. Then, at time $T-2$, all $n$ agents were 


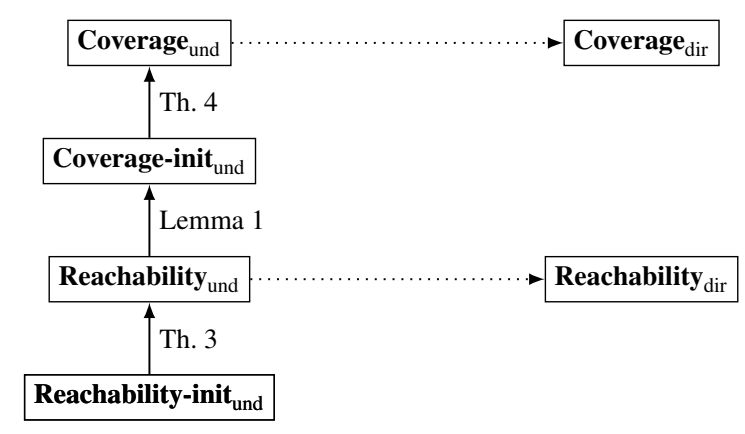

Fig. 6: Reductions of Section 5.

on the second layer $v_{i}^{2}$. In fact, the layer 4 must be empty at this point by the choice of $T$ and due to connectivity edges (and note also that there are no self-loops on the third layer). But since each node $v_{i}^{2}$ is only connected to $t_{i}$, at time $T-2$ the other $n$ agent must be at configuration $t$. This concludes the proof.

\section{Theorem 4 Coverage $_{\text {und }}$ is PSPACE-complete.}

Proof The upper bound comes from Proposition 1. We present the lower bound, which is by reduction from Coverage-init ${ }_{\text {und }}$. We map a Coverage-init und -instance $(G, n, s)$ to the Coverage und $^{\text {-instance }}\left(G^{\prime}, n+2\right)$ of where $G^{\prime}$ is defined as in Figure 4 (ignoring vertices $t_{1}, \ldots, t_{n}$ ). The formal description is given in the proof of Theorem 3.

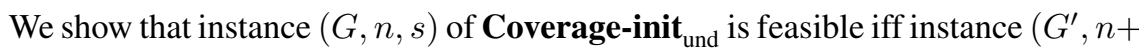
2 ) of Coverage und $_{\text {in }}$ is feasible. The proof is very similar to the proof of Theorem 3. The first $n$ agents are used for the execution in $G$ while the two others operate in the gadget.

$(\Rightarrow)$ If $G$ can be covered starting from $s$ then in $G^{\prime}$, the agents can first reach $s$ as described in proof of Theorem 3, then follow the same plan to cover the graph, and execute the plan in reverse to come back to $s$ and then back to $B^{\prime}$. Meanwhile, the two others reach $t_{n+1}$ and $t_{n+2}$ loops there and come back to $B^{\prime}$.

$(\Leftarrow)$ Assume there is a covering execution in $G^{\prime}$ from base $B^{\prime}$. We already proved in Theorem 3 (Fact 1) that from configuration $\left\langle B^{\prime}, \ldots, B^{\prime}\right\rangle$ the agents necessarily go through configuration $\left\langle s_{1}, \ldots, s_{n}, t_{n+1}, t_{n+2}\right\rangle$ to go in $G$ and that the first $n$ agents move in and out of $G$ at the same step. Hence, the execution in $G^{\prime}$, minus the steps in the gadget, can be reproduced in $G$.

We conclude this section by depicting, in Figure 6, the reductions used and the proof scheme. The dotted arrows represent the unmentioned corollaries.

\section{Sight-Moveable Topological Graphs}

The main challenge in deciding whether there exists a connected plan is to verify that the given number of agents can visit a node of the graph while staying connected. 


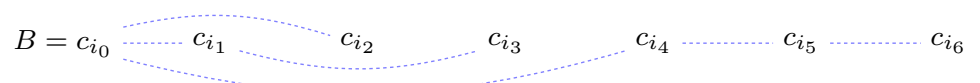

Fig. 7: Example of an ordering of nodes in $V^{\prime}=\left\{c_{1}, \ldots, c_{n}, B\right\}$.

Indeed, to visit a location connected to, say, the base, an agent might have to rely on multiple other agents. Hence, if we can guarantee that whenever two locations are connected we can move an agent from one to the other without the need of an extra relay, the problem becomes "easy". This assumption underlies the definition of sight-moveable topological graphs. Interestingly, the unbounded decision problems Reachability $_{\mathrm{sm}}$ and Coverage sm $_{\mathrm{sm}}$ are in LOGSPACE and NLOGSPACE, respectively. Unfortunately, the bounded version bReachability $_{\mathrm{sm}}$ is NP-complete. At the end of the section, we discuss a relaxation method based on this class of graphs.

\subsection{Upper Bounds}

Let us call USTCONN (resp. STCONN) the problem of determining whether two nodes of a given undirected (resp. directed) graph are connected. The algorithms presented in this section rely on the following complexity result:

\section{Theorem 5 ([42]) USTCONN is in LOGSPACE.}

\section{Proposition 3 Reachability $_{\mathrm{sm}}$ is in LOGSPACE.}

Proof Let us define the problem UCONN as that of checking whether a given undirected graph is connected. By Theorem 5, this problem is in LOGSPACE since it suffices to check the connectivity between all pairs of nodes in LOGSPACE.

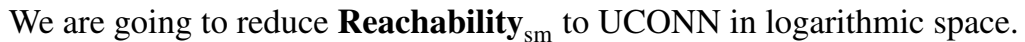

Let $G=\left\langle V, E_{m}, E_{c}\right\rangle$ a sight-moveable topological graph and $c$ a configuration. Let $V^{\prime}=\left\{c_{1}, \ldots, c_{n}, B\right\}$. We show that the configuration $c$ is reachable iff the restriction of $\mathfrak{G}^{\prime}:=\left(V, E_{c}\right)$ to the nodes in $V^{\prime}$ is a connected graph. It is clear that this condition is necessary since if $\mathfrak{G}^{\prime}$ is not connected then the agents cannot occupy configuration $c$.

Conversely, assume that $\mathfrak{G}^{\prime}$ is connected. Then, let us order the nodes $B, c_{1}, \ldots, c_{n}$ into $c_{i_{0}}, c_{i_{1}}, c_{i_{2}}, \ldots, c_{i_{n}}$ with $c_{i_{0}}=B$, such that for all $1 \leq j \leq n, c_{i_{j}}$ is connected to some $c_{i_{k}}$ with $0 \leq k<j$; such an order exists, and can be obtained by breadth-first search in a $E_{c}$-spanning tree of $V^{\prime}$ at root $B$, see Figure 7 for an example.

We then construct an execution for reaching the configuration $c$ as follows. The construction is by induction on $0 \leq j \leq n$ : at step $j$, the first $j$ agents occupy nodes $c_{i_{1}}, \ldots, c_{i_{j}}$. The base case $j=0$ is the empty execution. For $j \leq 1$, let $k<j$ such that $\left(c_{i_{k}}, c_{i_{j}}\right) \in E_{c}$. We send the $j$-th agent to $c_{i_{k}}$ following the path that has been constructed earlier, by induction. We then move that agent to $c_{i_{j}}$ following a path that stays connected with $c_{i_{j}}$, which exists by the sight-moveable property.

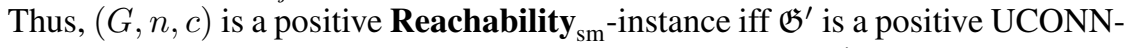
instance. The reduction is in logarithmic space: we compute $\mathfrak{G}^{\prime}$ by enumerating all 
$E_{c}$-edges $(u, v)$ in $G$, and we output $(u, v)$ when $u, v \in V^{\prime}$. We recall that we only take into account the working memory for computing $\mathfrak{G}^{\prime}$; the output $-\mathfrak{G}^{\prime}$ itself - is not taken into account in the used space (see e.g. [50], Ch. 8, Def. 8.21).

Before giving the complexity of Coverage $_{\mathrm{sm}}$, we need the following intermediary result. Let us call Bounded-USTCONN the following problem: given an undirected graph $\mathfrak{G}$, two nodes $s, t$, an integer $n$, decide whether there is a path of length at most $n$ from $s$ to $t$ in $G$. Note that, whatever the encoding of $n$ is, the problem BoundedUSTCONN can be decided in logarithmic space.

\section{Lemma 2 Bounded-USTCONN is in NLOGSPACE.}

Proof We reduce Bounded-USTCONN to STCONN in logarithmic space as follows. From a Bounded-USTCONN instance $(\mathfrak{G}, s, t, n)$ we construct in logarithmic space a STCONN instance $\left(\mathfrak{G}^{\prime}, s^{\prime}, t^{\prime}\right)$ :

1. The nodes of $\mathfrak{G}^{\prime}$ are pairs $(v, j)$ where $v$ is a node of $\mathfrak{G}$ and $j \in\{0,1, \ldots, m\}$ where $m$ is the minimum of $n$ and the number of nodes of $\mathfrak{G}$.

2. The graph $\mathfrak{G}^{\prime}$ contains an edge between $(v, j)$ and $\left(v^{\prime}, j+1\right)$ when there is an edge between $v$ and $v^{\prime}$ in $\mathfrak{G}$ or when $v=v^{\prime}$;

3. $s^{\prime}=(s, 0)$ and $t^{\prime}=(t, n)$.

The STCONN instance $\left(\mathfrak{G}^{\prime}, s^{\prime}, t^{\prime}\right)$ can be computed in log-space. Step 1 requires to store the current node $v$ of $\mathfrak{G}^{\prime}$ to be processed and an integer $j$ written in binary, that is of size $\log m$. Importantly, as $m$ is smaller than the number of nodes in $\mathfrak{G}^{\prime}$, the representation of $m$ is logarithmic in the size of the input. For this reason, note that the overall spatial complexity does not change even if the encoding of $n$ is in binary.

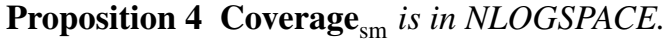

Proof Let $G=\left\langle V, E_{m}, E_{c}\right\rangle$ be a sight-moveable topological graph and $n$ an integer written in unary. We prove that for all vertices $v$, there is a path of length at most $n$ from $v$ to the base $B$ in the communication graph iff $(G, n)$ is a positive instance of Coverage $_{\mathrm{sm}}$. One direction is obvious: if $(G, n)$ is a positive instance, then all vertices must within a distance of at most $n$ from the base in the communication graph; otherwise no connected configuration can visit that vertex. Assume that all vertices are within a distance of $n$ from the base. For any vertex $v$, consider path $v_{1}, \ldots, v_{k}$ in the communication graph, with $v_{1}=B, v_{k}=B$, and such that $\left(v_{i}, v_{i+1}\right) \in E_{c}$. We apply the construction of Proposition 3 to build an execution from configuration $B^{n}$ to some configuration where $k$ agents occupy $\left\{v_{1}, \ldots, v_{k}\right\}$, and others stay at $B$. We now extend this execution to "roll back" so that all agents return to the base. We first let the agent at $v_{k}$, go to $v_{k-1}$ : this is possible by the sight-moveable property and since $\left(v_{k}, v_{k-1}\right) \in E_{c}$. Then the two agents together move to $v_{k-2}$, and so on, until all come back to $B$. We can now combine these executions to cover all vertices, and let agents end in the base.

Thus, the algorithm consists in checking sequentially, for all $v$, that $\left(\left(V, E_{c}\right), v, B, n\right)$ is a positive instance of Bounded-USTCONN. Hence, we obtain a non-deterministic algorithm in logarithmic space to decide Coverage $_{\mathrm{sm}}$. 


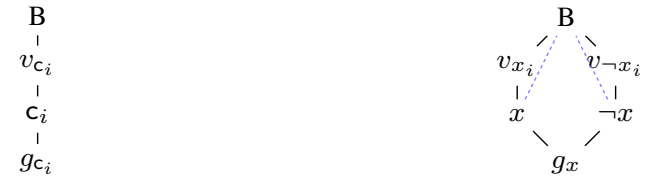

(a) Clause gadget

(b) Variable gadget

Fig. 8: Gadgets for reduction of 3SAT into bReachability ${ }_{\mathrm{sm}}$

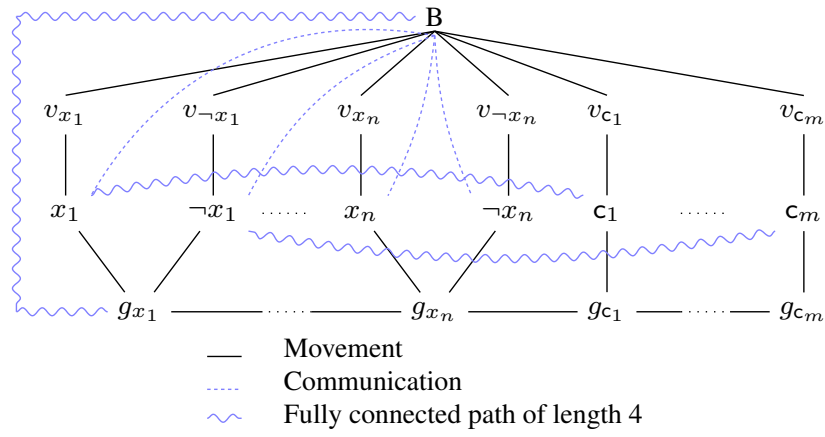

Fig. 9: Reduction of 3SAT into bReachability ${ }_{\mathrm{sm}}$. Communication edges implied by movement edges are not displayed. The variable $x_{1}$ is present in the clause $c_{1}$ and $c_{n}$.

\subsection{Lower Bounds}

We now focus on the NP lower bound of $\mathbf{b R e a c h a b i l i t y ~}_{\mathrm{sm}}$.

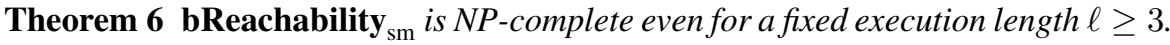

Proof The upper bound comes from Proposition 2. The lower bound proof is by polynomial time reduction from 3-SAT problem (see [28]). Given a 3-SAT instance, set of clauses $c_{1}, \ldots, c_{m}$ with variables $x_{1}, \ldots, x_{n}$, we describe the construction of an instance $(G, k, c)$ of bReachability sm $_{\text {sm }}$ with $k=n+m$ agents.

The topological graph $G=\left\langle V, E_{m}, E_{c}\right\rangle$ is constructed as follows. We start by placing the base $B$ from which the agents start their mission.

Please recall that in a sight-moveable graph all movements edges are also communication edges in the construction below even if not explicitly stated.

For each variable $x$, we construct a gadget composed of 5 nodes connected to the base depicted in Figure $8 \mathrm{~b}$ : nodes $x_{i}, \neg x_{i}$, staging nodes $v_{x_{i}}, v_{\neg x_{i}}$ and a goal node $g_{x_{i}}$. We add movement edges from $B$ to $v_{x_{i}}$, from $v_{x_{i}}$ to $x$ and from $x$ to $g_{x_{i}}$ (resp. from $B$ to $v_{\neg x_{i}}$, from $v_{\neg x_{i}}$ to $\neg x_{i}$ and from $\neg x_{i}$ to $g_{x_{i}}$ ). As for the communication, the node $x_{i}$ (res. $\neg x_{i}$ ) communicates with the base.

For each clause $c_{j}$, we construct a gadget composed of 3 nodes depicted in Figure 8a. We create a node $\mathrm{c}_{j}$, a staging node $v_{\mathrm{c}_{j}}$ and a goal node $g_{\mathrm{c}_{j}}$. We add movement edges from $B$ to $v_{c}$, from $v_{\mathrm{c}_{j}}$ to $\mathrm{c}_{j}$ and from $\mathrm{c}_{j}$ to $g_{\mathrm{c}_{j}}$. The communication between a clause $c_{j}$ and a literal $x_{i}$ or $\neg x_{i}$ is dictated by the existence of the literal in the clause. We do not use direct communication edges because the obtained topological graph 
should be sight-moveable. Instead, we use fully connected paths of length 4 . Such a path between - let say $-x_{i}$ and $c_{j}$ consists three intermediate nodes $p_{i j}^{1}, p_{i j}^{2}, p_{i j}^{3}$, such that there is a path made up of movement edges from $x_{i}$ to $c_{j}$, passing throw $p_{i j}^{1}, p_{i j}^{2}$, $p_{i j}^{3}$. Furthermore, we suppose that the nodes $x_{i}, p_{i j}^{1}, p_{i j}^{2}, p_{i j}^{3}$ and $\mathrm{c}_{j}$ form a clique w.r.t. to the communication edges. Now, there is a fully connected path of length 4 between $x_{i}$ and $\mathrm{c}_{j}$ if and only if $x_{i} \in \mathrm{c}_{j}$; and there is a fully connected path of length 4 between $\neg x_{i}$ and $\mathrm{c}_{j}$ if and only if $\neg x_{i} \in \mathrm{c}_{j}$.

We add movement edges from $g_{x_{i}}$ to $g_{x_{i+1}}$, and from $g_{\mathrm{c}_{j}}$ to $g_{\mathrm{c}_{i+1}}$ for all $1 \leq i<$ $n$, as well as from $g_{x_{n}}$ to $g_{\mathrm{c}_{1}}$. Last, we add a fully connected path of length 4 from $g_{x_{1}}$ to the base such that $\left(g_{x_{1}}, B\right) \in E_{c}$, in the sense that all nodes of this path have communication edges between them. This translation is polynomial in the number of clauses and variables. The construction is depicted in Figure 9. The snake-like path from $g_{x_{1}}$ to $B$ is a fully connected path of length 4 .

From a 3-SAT instance, one can construct the graph $G$ and ask for an execution of length 3 to reach the configuration $\left\langle g_{x_{1}}, \ldots, g_{x_{n}}, g_{\mathrm{c}_{1}}, \ldots, g_{\mathrm{c}_{m}}\right\rangle$.

Formally, the topologic graph $G=\left\langle V, E_{m}, E_{c}\right\rangle$ is defined by:

$$
\begin{aligned}
-V & :=\left\{B, v_{x_{1}}, v_{\neg x_{1}}, \ldots, v_{x_{n}}, v_{\neg x_{n}}, v_{\mathrm{c}_{1}}, \ldots, v_{\mathrm{c}_{m}}\right\} \\
& \cup\left\{g_{x_{1}}, \ldots, g_{x_{n}}, g_{\mathrm{c}_{1}}, \ldots, g_{\mathrm{c}_{m}}\right\} \\
& \cup\left\{p_{B}^{1}, p_{B}^{2}, p_{B}^{3}\right\} \cup\left\{p_{i j}^{1}, p_{i j}^{2}, p_{i j}^{3} \mid i \in\{1, \ldots, n\}, j \in\{1, \ldots, m\}\right\}
\end{aligned}
$$

- $E_{m}$ is the symmetric closure of

$$
\begin{aligned}
&\left\{\left(B, v_{x_{1}}\right),\left(B, v_{\neg x_{1}}\right), \ldots,\left(B, v_{x_{n}}\right),\left(B, v_{\neg x_{n}}\right),\left(B, v_{\mathrm{c}_{1}}\right),\left(B, v_{\mathrm{c}_{1} x_{m}}\right)\right\} \\
& \cup\left\{\left(v_{x_{1}}, x_{1}\right),\left(v_{\neg x_{1}}, \neg x_{1}\right), \ldots\left(v_{x_{n}}, x_{n}\right),\left(v_{\neg x_{n}}, \neg x_{n}\right)\right\} \\
& \cup\left\{\left(v_{\mathrm{c}_{1}}, \mathrm{c}_{1}\right), \ldots,\left(v_{\mathrm{c}_{m}}, \mathrm{c}_{m}\right)\right\} \\
& \cup\left.\cup\left(x_{1}, g_{x_{1}}\right),\left(\neg x_{1}, g_{x_{1}}\right), \ldots,\left(x_{n}, g_{x_{n}}\right),\left(\neg x_{n}, g_{x_{n}}\right)\right\} \\
& \cup\left\{\left(\mathrm{c}_{1}, g_{\mathrm{c}_{1}}\right), \ldots,\left(\mathrm{c}_{m}, g_{\mathrm{c}_{m}}\right)\right\} \\
& \cup\left\{\left(g_{x_{1}}, g_{x_{2}}\right), \ldots\left(g_{x_{n-1}}, g_{x_{n}}\right),\left(g_{x_{n}}, g_{\mathrm{c}_{1}}\right),\left(g_{\mathrm{c}_{1}}, g_{\mathrm{c}_{2}}\right), \ldots,\left(g_{\mathrm{c}_{m-1}}, g_{\mathrm{c}_{m}}\right\}\right. \\
& \cup\left\{\left(B, p_{B}^{1}\right),\left(p_{B}^{1}, p_{B}^{2}\right),\left(p_{B}^{2}, p_{B}^{3}\right),\left(p_{B}^{3}, g_{x_{1}}\right)\right\} \\
& \cup\left\{\left(x_{i}, p_{i j}^{1}\right),\left(p_{i j}^{1}, p_{i j}^{2}\right),\left(p_{i j}^{2}, p_{i j}^{3}\right),\left(p_{i j}^{3}, \mathrm{c}_{j}\right) \mid x_{i} \in \mathrm{c}_{j}\right\} \\
& \cup\left\{\left(\neg x_{i}, p_{i j}^{1}\right),\left(p_{i j}^{1}, p_{i j}^{2}\right),\left(p_{i j}^{2}, p_{i j}^{3}\right),\left(p_{i j}^{3}, \mathrm{c}_{j}\right) \mid \neg x_{i} \in \mathrm{c}_{j}\right\}
\end{aligned}
$$

$-E_{c}$ is the symmetric closure of

$$
\begin{aligned}
& E_{m} \cup\left\{\left(B, x_{1}\right),\left(B, \neg x_{1}\right), \ldots,\left(B, x_{n}\right),\left(B, \neg x_{n}\right)\right\} \\
& \cup\left\{\begin{array}{l}
\left(B, p_{B}^{1}\right),\left(p_{B}^{1}, p_{B}^{2}\right),\left(p_{B}^{2}, p_{B}^{3}\right),\left(p_{B}^{3}, g_{x_{1}}\right), \\
\left(B, p_{B}^{2}\right),\left(p_{B}^{1}, p_{B}^{3}\right),\left(p_{B}^{2}, g_{x_{1}}\right) \\
\left(B, p_{B}^{3}\right),\left(p_{B}^{1}, g_{x_{1}}\right),\left(B, g_{x_{1}}\right)
\end{array}\right\} \\
& \cup\left\{\begin{array}{l}
\left(x_{i}, p_{i j}^{1}\right),\left(p_{i j}^{1}, p_{i j}^{2}\right),\left(p_{i j}^{2}, p_{i j}^{3}\right),\left(p_{i j}^{3}, \mathrm{c}_{j}\right), \\
\left(x_{i}, p_{i j}^{2}\right),\left(p_{i j}^{1}, p_{i j}^{3}\right),\left(p_{i j}^{2}, \mathrm{c}_{j}\right) \\
\left(x_{i}, p_{i j}^{3}\right),\left(\mathrm{c}_{i j}^{1}, \mathrm{c}_{j}\right),\left(x_{i}, \mathrm{c}_{j}\right)
\end{array}\right\} \\
& \cup\left\{\begin{array}{l}
\left(\neg x_{i}, p_{i j}^{1}\right),\left(p_{i j}^{1}, p_{i j}^{2}\right),\left(p_{i j}^{2}, p_{i j}^{3}\right),\left(p_{i j}^{3}, \mathrm{c}_{j}\right), \mid \neg x_{i} \in \mathrm{c}_{j} \\
\left(\neg x_{i}, p_{i j}^{2}\right),\left(p_{i j}^{1}, p_{i j}^{3}\right),\left(p_{i j}^{2}, \mathrm{c}_{j}\right) \\
\left(\neg x_{i}, p_{i j}^{3}\right),\left(p_{i j}^{1}, \mathrm{c}_{j}\right),\left(\neg x_{i}, \mathrm{c}_{j}\right)
\end{array}\right\} ;
\end{aligned}
$$

where nodes $p_{\bullet}^{k}$ are the intermediate nodes in the fully connected paths.

Fact $3 G$ is a sight-moveable topological graph. 
Proof Concerning the communication between the base $B$ and the nodes $x_{i}$ (resp. $\neg x_{i}$ ), a path does exist under the communication of $B$ to reach $x_{i}$ (resp. $\neg x_{i}$ ), due to communication induced by the movement. For the other communication edges, they are part of full connected paths which guarantee the sight-moveable condition. This ends the proof of Fact 3.

Now let us prove that a 3-SAT instance is satisfiable iff there exists an execution of at most 3 steps in the graph $G$.

$(\Rightarrow)$ We show that if a 3-SAT instance is satisfiable then there exists an execution of at most 3 steps in the graph $G$ built from it. Let val be a truth assignment which satisfies the instance. Recall that there are $n+m$ agents. The first step of the execution consists in moving an agent in each $v_{c_{i}}$, and for each variable $x_{i}$, moving one agent to $v_{x_{i}}$ if the $\operatorname{val}\left(x_{i}\right)=1$ and to $v_{\neg x_{i}}$ otherwise. Note that all staging nodes communicate with $B$.

In the second step, all agents progress to their unique successors other than $B$. While all nodes $x_{i}$ and $\neg x_{i}$ are connected to $B$, a node $c_{i}$ is connected to $B$ if and only if there is an agent in one of its literals. This is the case since val satisfies the formula. In the third step of the execution, agents go to states $g_{x_{i}}$ and $g_{c_{i}}$. Here, the connection with the base is ensured since $g_{x_{1}}$ is connected to it, and $g_{x_{2}}$ is connected to $g_{x_{1}}, g_{x_{3}}$ is connected to $g_{x_{2}}$ and so on.

This execution is thus a solution of bReachability $\mathbf{s}_{\mathrm{sm}}$ with bound $\ell=3$.

$(\Leftarrow)$ We show that if in the graph $G$ there exists an execution of at most 3 steps constructed from a 3-SAT instance, then the instance is satisfiable. Let us assume that we have an execution $e$ of at most 3 steps with the last configuration being $\left\langle g_{x_{1}}, \ldots, g_{x_{n}}, g_{\mathrm{c}_{1}}, \ldots, g_{\mathrm{c}_{m}}\right\rangle$.

The only shortest path from $B$ to $g_{c_{i}}$ is of length 3 and goes through $v_{c_{i}}$. For states $g_{x_{i}}$, the only shortest paths are also of length 3 and go through either $v_{x_{i}}$ or $v_{\neg x_{i}}$. Thus, in order to reach the given target configuration, at the initial step, agents must cover the states $v_{c_{i}}$ and either $v_{x_{i}}$ or $v_{\neg x_{i}}$ for all $i, j$. At the second step, following the above mentioned shortest paths, agents will be at states $c_{i}$ and either $x_{i}$ or $\neg x_{i}$ depending on the staging nodes they were occupying. The last step is the target configuration. Since the agents are connected at the second, it follows that for each clause $\mathrm{c}_{j}$, the state corresponding to some literal of $\mathrm{c}_{j}$ is occupied by an agent. Thus, the valuation on variables encoded by the choices of the agents satisfies the 3-SAT instance.

\subsection{Relaxation}

For unbounded reachability and coverage, it seems tempting to take advantage of efficient algorithms on sight-moveable topological graphs (Proposition 3 and 4). In this subsection, we propose a transformation of a topological graph into a sight-moveable sub-graph. This transformation leads to a relaxation method: a solution found in the obtained sight-moveable topological graph still holds in the original graph.

This transformation requires the original graph to already satisfy properties 1 and 2 of Definition 3. If not, (1) we remove movement edges $\left(v, v^{\prime}\right) \in E_{m}$ that are not in $E_{c},(2)$ we remove all nodes without a self-loop. 


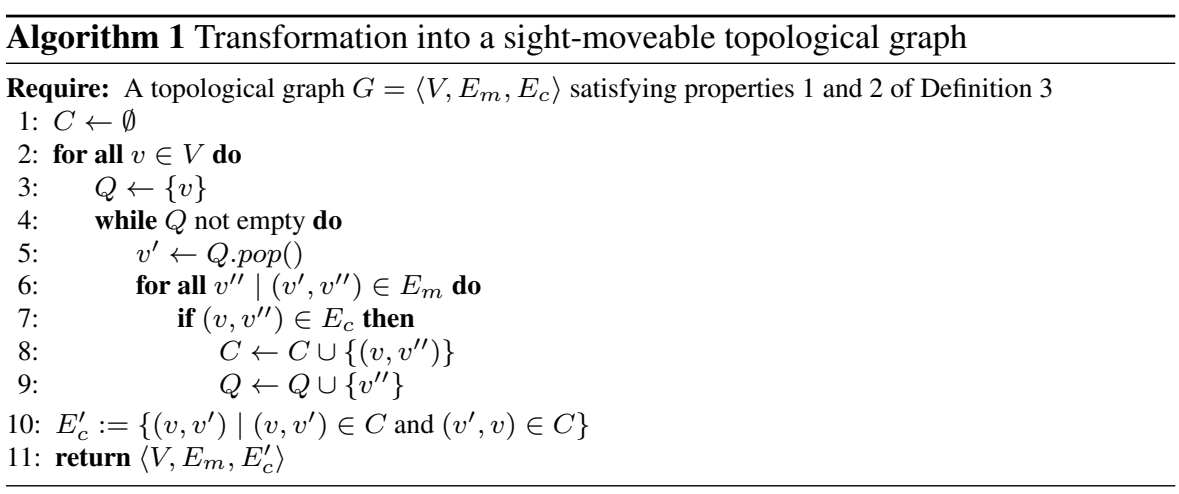

The simple transformation, given in Algorithm 1, prunes the communication edges which do not respect the property 3 of sight-moveable topological graphs, described in Definition 3. We now show this transformation outputs a sight-moveable topological graph.

\section{Theorem 7 Algorithm 1 constructs a sight-moveable topological graph.}

For simplicity, we say that a node $v$ is sight-moveable to a node $v^{\prime}$ iff there exists a sequence $\rho=\left\langle\rho_{1}, \ldots, \rho_{n}\right\rangle$ of nodes such that $v=\rho_{1}, v^{\prime}=\rho_{n},\left(v, \rho_{i}\right) \in E_{c}$ and $\left(\rho_{i}, \rho_{i+1}\right) \in E_{m}$ for all $i \in\{1, \ldots, n-1\}$. Algorithm 1 consists in running a breadth-first search from each vertex, and marking all reachable vertices $v^{\prime}$ to which $v$ is sight-moveable. The communication edges are kept if the sight-moveable property was shown to hold in both directions.

Proof First, given a node $v$, we show that the queue $Q$ contains only nodes that $v$ is sight-moveable to.

Invariant Given $v$, let $I_{v}$ : for all $v^{\prime} \in Q, v$ is sight-moveable to $v^{\prime}$.

Proof Before entering the while loop, at Line 3, the queue $Q$ contains only $v$. Hence, the Invariant $I_{v}$ is initially satisfied. Let us suppose that Invariant $I_{v}$ holds after an arbitrary number of loop iterations. If $Q$ is empty, Invariant $I_{v}$ holds. Otherwise, a node $v^{\prime}$ is popped out of $Q$. If a successor of $v^{\prime}$ communicates with $v$ then we add the successors of $v^{\prime}$ to $Q$. Invariant $I_{v}$ holds since $v$ is sight-moveable to $v^{\prime}$, by induction, and $\left(v, v^{\prime \prime}\right) \in C$.

We add a pair $\left(v, v^{\prime}\right)$ to $C$ iff $v^{\prime}$ was in $Q$ and $\left(v, v^{\prime}\right) \in E_{c}$. Hence, given Invariant $I_{v},\left(v, v^{\prime}\right) \in C$ iff $v$ is sight-moveable to $v^{\prime}$. Finally, $E_{c}^{\prime}$ is the set of pairs $\left(v, v^{\prime}\right)$ such that $\left(v, v^{\prime}\right) \in C$ and $\left(v^{\prime}, v\right) \in C$, that is $v$ is sight-moveable to $v^{\prime}$ and $v^{\prime}$ is sightmoveable to $v$. Therefore, the graph returned by Algorithm 1 is sight-moveable.

This relaxation offers the opportunity to verify efficiently if a topological graph contains a solution. It is worth noting that the original topological graph might admit a solution, while no solution is found by the relaxation.

We illustrate the usage of the relaxation on the following maps, depicted in Figure 10, previously used by Tateo et al., in [54], for the study of their algorithms. 


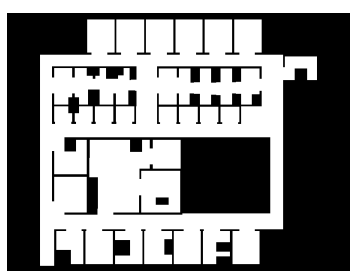

(a) Office

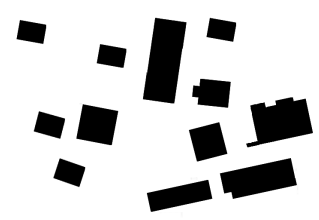

(b) Open

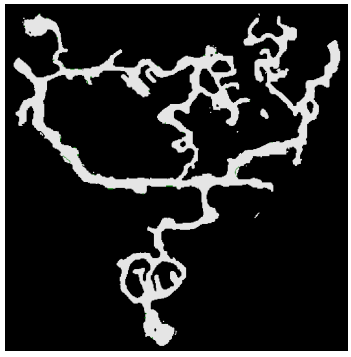

(c) Coast

Fig. 10: Maps.

\begin{tabular}{l||c|c|c||c}
\multirow{2}{*}{\multicolumn{1}{c||}{ Map }} & \multicolumn{3}{c||}{ Original Graph } & Relaxation \\
\cline { 2 - 5 } & $|V|$ & $\left|E_{m}\right|$ & $\left|E_{c}\right|$ & $\left|E_{c}\right|$ \\
\hline \hline Office & 1669 & 5618 & 277059 & 227155 \\
\hline Open & 2421 & 9114 & 295155 & 294503 \\
\hline Coast & 5184 & 20448 & 580644 & 580644
\end{tabular}

Fig. 11: Characteristics of the discretized graphs and their relaxations.

We use a similar discretization of the maps, i.e. the nodes of the graph are obtained by cells of $11 \times 11$ pixels for Office and $13 \times 13$ for Open, and we assume the communication range to be of 100 pixels. In addition, we use the same discretization with cells of $15 \times 15$ pixels for Coast, a map from the Benchmarks for Grid-Based Pathfinding [53], with an identical communication range.

We show, in Figure 11, the size of each component of the graphs obtained after discretization and the impact of the relaxation. The relaxation of Office removes up to $18 \%$ of the communication edges. We can observe that most of the communication trough the walls, allowed by the communication range, will be removed in the relaxation since the sight-moveable property cannot be ensured. In particular the large room in the center loses a lot of communication edges with its surrounding. However, the relaxation of Open removes only $0.2 \%$ of the communication due to its small number of obstacles. Finally, the relaxation does not remove any communication edges from the discretization made of Coast.

Intuitively, the relaxation removes communication links between two vertices which cannot be connected by maintaining communication. In fact, if a node communicates through an obstacle, then the sight-moveable property requires it to have a bypass path that remains connected. This is why, in the Office map, in particular at the borders of the central room, most communication links through walls are removed by 
the algorithm. But if the size of the obstacles is larger than the communication range, then there will be mostly no communication through obstacles, and it is unlikely that the relaxation will remove any communication edges: this can be observed on the Coast map where no edge is removed by the algorithm.

Thus, in open maps such as cities, forests etc., we expect that our relaxation removes a small number of edges and preserves the feasibility of our problems. It might however remove more communication edges, rendering the problems infeasible in indoor applications (inside of buildings, mazes, etc.). We leave the empirical evaluation of this relaxation for future work.

\section{Complete-Communication Topological Graphs}

The following result relies on the fact that the communication is complete.

Proposition 5 bReachability $_{\mathrm{cc}}$ is in NLOGSPACE.

Proof We refer to Lemma 2. Indeed, given a configuration $c$ and $\ell \in \mathbb{N}$, the straightforward iteration on the locations $c_{i}$ followed by the verification of a path of at most $\ell$ (given in unary) steps from $B$ to $c_{i}$ yields a sound and complete algorithm for bReachability $_{\mathrm{cc}}$.

Our NP lower bound proof of the bCoverage bc $_{\mathrm{c}}$ problem is by reduction from the grid Hamiltonian cycle (G-HC) problem which is the Hamiltonian cycle problem restricted to grid graphs and is NP-complete [27]. We use this particular version of the Hamiltonian cycle problem for simplicity of the proof. Futhermore, we obtain a lower bound on the bCoverage bc $_{\mathrm{c}}$ problem with a grid movement graph.

Theorem 8 Even restricted to grid graphs, bCoverage cc $_{\text {is }}$ is-complete for a fixed number of agents $n \geq 1$.

Proof The upper bound follows from Proposition 2.

We give a polynomial-time reduction from the $\mathbf{G}-\mathbf{H C}$ problem. Consider an instance of $\mathbf{G - H C}$ denoted $G=\langle V, E\rangle$.

Consider the sight-moveable topological graph $G^{\prime}=\left\langle V, E_{m}, E_{c}\right\rangle$ with undirected movement $E_{m}=E$ and $E_{c}=V \times V$ and associate a single agent and the

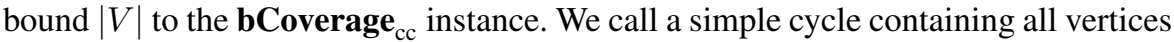
a tour. We prove that there exists a tour $t$ in $G$ iff there exists a covering execution of length $|V|$ in $G^{\prime}$.

$(\Rightarrow)$ Any tour of $G$ is a valid execution satisfying bCoverage bc $_{\mathrm{cc}}$ since the communication edges form a complete graph, and the bound is $|V|$.

$(\Leftarrow)$ Let us suppose that we have an execution of length $|V|$ which covers the graph $G^{\prime}$. The execution starts and ends at $B$ and visits all nodes in $|V|$ steps. Hence, the execution visits all nodes only once and is a cycle in the graph.

In Figure 12, we depicted the results obtained in this section and in Section 7. 


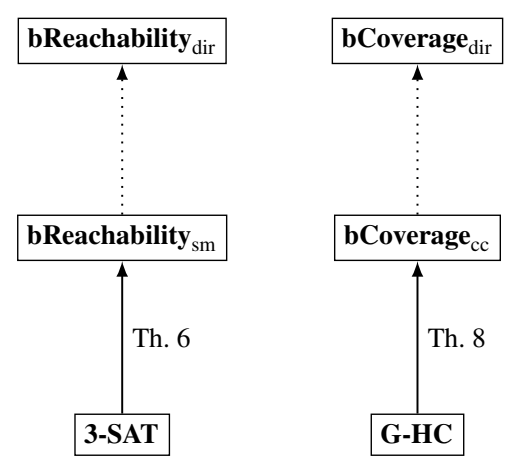

Fig. 12: Reductions of Sections 6 and 7

\section{Variants}

In this section, we introduce several variants and study their impact on the complexity.

\subsection{Bounded Reachability and Coverage with Binary Bounds}

Our membership NP proofs for the bounded versions of the reachability and coverage problems rely on the unary encoding of the bound given in input. It is relevant to investigate the impact of providing that bound in binary on the complexity of the problems.

We show that both problems bReachability dir $_{\text {and }}$ bReachability und $_{\text {with the }}$ bound $\ell$ written in binary are both PSPACE-complete. The membership to PSPACE can be shown as in Proposition 1: since a binary counter can be added to count up to $\ell$. For $\mathbf{b R e a c h a b i l i t y}_{\text {und }}$, the PSPACE-hardness follows from a reduction from Reachability $_{\text {und }}$, whose PSPACE-hardness is established in Theorem 3. Indeed, any instance of Reachability und $_{\text {can }}$ be reduced to bounded reachability with bound $\ell=$

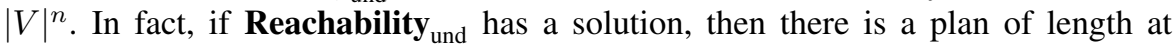
most $|V|^{n}$. Indeed, in the worst case, the agents must go through all configurations possible in the graph. This number can be computed in polynomial time and represented in binary. The same argument is used to show the PSPACE-hardness of bReachability $_{\text {dir }}$ by reduction from Reachability ${ }_{\text {dir }}$ whose PSPACE-hardness was proved in [54] (see Theorem 2).

Theorem 9 The variants of $\mathbf{b R e a c h a b i l i t y} \mathrm{dir}_{\mathrm{di}}$ and $\mathbf{b R e a c h a b i l i t y} \mathbf{y}_{\mathrm{und}}$ with $\ell$ written in binary are PSPACE-complete.

A similar argument can be used to show the PSPACE-hardness of bCoverage

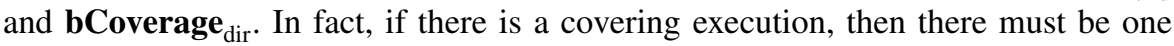
of length at most $|V|^{n} \times(|V|+1)$. Indeed, the agents must traverse at most the $|V|^{n}$ possible configurations to reach each node of the graph and go back to the base, which means an upper bound of $|V|^{n} \times(|V|+1)$ for the whole execution. This bound can be written in binary in polynomial space, so PSPACE-completeness also holds for these problems. 
Theorem 10 The variants of $\mathbf{b C o v e r a g e}_{\mathrm{dir}}$ and $\mathbf{b C o v e r a g e}_{\mathrm{und}}$ with $\ell$ written in binary are PSPACE-complete.

\subsection{Weighted Movement Graph}

An interesting extension is obtained by assigning costs to edges of the movement graph, and considering the bounded reachability problem with respect to the total cost of the execution.

Consider a weighted topological graph $G=\left\langle V, E_{m}, E_{c}\right.$, cost $\rangle$, where for each edge $\operatorname{cost}(e)$ is the cost of $e$, a positive integer.

One could consider several ways of aggregating the weights along executions. We consider the case where the weights correspond to travel times between vertices, and the goal is to minimize total travel time, which also implies minimizing battery usage in drone applications. We consider a synchronous setting where all agents synchronize at each configuration in the plan; therefore, the travel time between two configurations is the maximum of the weights of the edges along which agents travel. In other terms, we assume that agents wait for each other at each step of the execution.

Formally, for an execution $c_{0}, c_{1}, \ldots, c_{k}$, the total cost is defined as follows:

$$
\sum_{i=0}^{k-1} \max _{j \in\{1,2, \ldots, n\}} \operatorname{cost}\left(c_{i}[j], c_{i+1}[j]\right),
$$

where $c_{i}[j]$ denotes the vertex occupied by agent $j$ at configuration $c_{i}$. Assuming binary encoding of all weights, we are interested in checking the existence of an execution of bounded cost, and that of a covering execution of bounded cost.

When weights are encoded in unary, both reachability and coverage problems are NP-hard since when all costs are equal to 1 , then the problems are identical to bReachability and bCoverage, respectively. Since all weights are natural numbers, the length of the execution is not more than the cost bound given in input. Thus, similarly to Proposition 2, one can guess an execution and bound its length with a polynomial number of guesses.

Theorem 11 The weighted variants of bReachability, bCoverage on directed and undirected topological graphs with unary encoding are NP-complete.

When the weights are encoded in binary, then the problems are PSPACE-hard as shown in Section 8.1; and the PSPACE algorithms can be extended to establish PSPACE-completeness.

Theorem 12 The weighted variants of bReachability, bCoverage on directed and undirected topological graphs with binary encoding are PSPACE-complete.

\subsection{Bounded Disconnection}

Another extension consists in allowing the agents to be disconnected for a bounded number of steps along the execution. Such a feature can be desirable in order to allow 
the agents to reach difficult locations where communication cannot be guaranteed. By bounding the disconnection time, the connection with the base is only disturbed temporarily.

This extension is also PSPACE-complete. In fact, our membership results can easily be extended both for reachability and coverage problems. For PSPACE-hardness, observe that when the allowed bound is 0 , the problems become identical to our setting, so all hardness proofs carry over.

\subsection{Collisions}

Collision constraints consist in disallowing several agents to share the same location at a given time, and provide an interesting extension of our setting. In our case, we allow several agents to be at the base but not at other nodes.

The PSPACE-completeness results hold by using the same reductions used for the proof of Theorems 3 and 4 from the collision-free variant problem of Tateo et al. [54]. Indeed, the reductions gadgets used are collision-free, and thus we obtain:

Theorem 13 The variants of Reachability $_{\mathrm{und}}$, Reachability ${ }_{\mathrm{dir}}$, Coverage $_{\mathrm{und}}$ and Coverage $_{\mathrm{dir}}$ with collision constraints are PSPACE-complete.

We can show that when the topological graph is sight-moveable, then the complexity of deciding the reachability and coverage without allowing collisions is unchanged. We use the same membership proof of Proposition 3 and 4. Indeed, a positive instance of Reachability sm $_{\mathrm{sm}}$ (resp. Coverage sm $_{\mathrm{sm}}$ ) is a positive instance of Reachability $\mathbf{~}_{\mathrm{sm}}$ (resp. Coverage $_{\mathrm{sm}}$ ) with collision constraints. In order to obtain a collision-free execution, it suffices to modify as follows: we consider an ordering, as depicted in Figure 7, and consider each branch one by one. For each branch, we dispatch first the agent with the furthest target from the base, followed by the second furthest agent and so on. This yields a collision-free execution for Reachability sm $_{\mathrm{sm}}$. Coverage $\mathbf{s}_{\mathrm{sm}}$ can be solved by a repeated application of Reachability $\mathbf{y}_{\mathrm{sm}}$. Observe that Reachability $\mathbf{y}_{\mathrm{cc}}$ and Coverage $_{\mathrm{cc}}$ can use the same algorithms.

Theorem 14 The variants of Reachability sm $_{\mathrm{sm}}$ Reachability $_{\mathrm{cc}}$ (resp. Coverage . $_{\mathrm{sm}}$ and Coverage $_{\mathrm{cc}}$ ) with collision constraints are in LOGSPACE (resp. NLOGSPACE).

Regarding the bounded versions, the membership of Proposition 2 hold without allowing collisions. Indeed, checking if the path contains a collision can be done in polynomial-time. The lower bound can be showed by reduction from the MAPF problem to bReachability bc $_{\text {. }}$. Additionally, the proof of Theorem 8 holds without allowing collisions given that the proof holds for a single agent.

Theorem 15 The variants of $\mathbf{b R e a c h a b i l i t y}_{\star}$ and $\mathbf{b C o v e r a g e}_{\star}$ without collisions are $N P$-complete for all $\star \in\{$ und, dir, sm, cc $\}$. 


\subsection{Planar Movement Graphs}

One could wonder whether the complexity of these problems change when restricted to topological graphs whose movement graphs are planar. This is an interesting question since in targeted applications the graphs are indeed planar; and some problems are known to become easier on planar graphs (e.g. the shortest path computation [30]). In this section, we show that our reductions proving the PSPACE-hardness

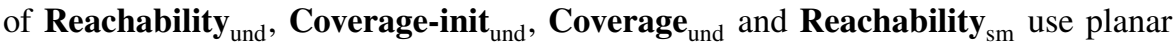
graphs, which means that these hardness results hold even when the input is restricted to planar graphs.

Actually, the proof of Reachability-init ${ }_{\text {und }}$, in [54], uses a planar movement graph. They even prove a stronger result: the reachability problem is PSPACE-hard even for graphs that are disjoint unions of paths ${ }^{2}$.

Let us consider an instance $\left(G_{\text {Reach }_{\text {init }}}, B, s, t\right)$ of Reachability-init ${ }_{\text {und }}$ where $G_{\text {Reach }_{\text {init }}}$ is a topological graph that is a disjoint union of paths (depicted in the middle of Figure 13), $B$ is its base, $s=\left(s_{1}, \ldots, s_{n}\right)$ is initial configuration for $n$ agents, depicted at the bottom of $G_{\text {Reach }}$ init in Figure 13 and $t=\left(t_{1}, \ldots, t_{n}\right)$ is the target configuration (the target nodes $t_{1}, \ldots, t_{n}$ are in $G_{\text {Reach }}$ init $_{\text {as defined in [54] }}$ but are not displayed in the figure).

Then as shown in Figure 13, the gadgets we proposed in this article can all be arranged to obtain planar graphs as well.

1. The gadget given in Figure 4, used in the proof of Theorem 3 is a planar graph; in fact, it can be arranged, to obtain $G_{\text {Reach }}$ (in Fig. 13) whose base is $B^{\prime}$ and is designed for $n+2$ agents. This proves that Reachability und $_{\text {is PSPCE-hard on }}$ planar graphs.

2. We consider now the reduction of Figure 5 used in proof of Lemma 1, which contains $G_{\text {Reach }}$. This reduction, named $G_{\text {Cover }_{\text {init }}}$ in Figure 13 is also planar. The base of this graph is still $B^{\prime}$, and it is designed for $n+2+n+2=2 n+4$ agents. In this reduction, the first $n+2$ agents start in $B^{\prime}$ while the others start in $v_{1}^{1}, \ldots, v_{n+2}^{1}$. Indeed, the latter gadget is disconnected from the rest of the graph, except that the node $v_{1}^{4}$ has a movement edge to all nodes in the graph. One can observe that a movement edge can be created from $v_{1}^{4}$ to all nodes in the graph $G_{\text {Reach }}$, while keeping the construction planar, thanks to the particular structure of $G_{\text {Reach }}$. This shows that Coverage-init ${ }_{\text {und }}$ is PSPACE-hard on planar graphs.

3. Finally, we can add the gadget of Figure 4 used in proof of Theorem 4 , shown in the top part of Figure 13. We thus obtain $G_{\text {Cover }}$ designed for $2 n+4+2=2 n+6$ agents starting at the base $B^{\prime \prime}$, which is the reduction of Theorem 4 . The obtained graph is also planar. This shows that Coverage $_{\text {und }}$ is PSPACE-hard on planar graphs.

To sum up:

2 Their reduction is from a problem called the reconfiguration problem on constraint graphs. The topological graph computed from a constraint graph contains single nodes $(\bigcirc)$, paths of length 3 (which we denote by $\bigcirc$ ) and a single path graph of length $|E|$ (denoted by $\square+\cdots)$, with $E$ the set of edges in the constraint graph. 


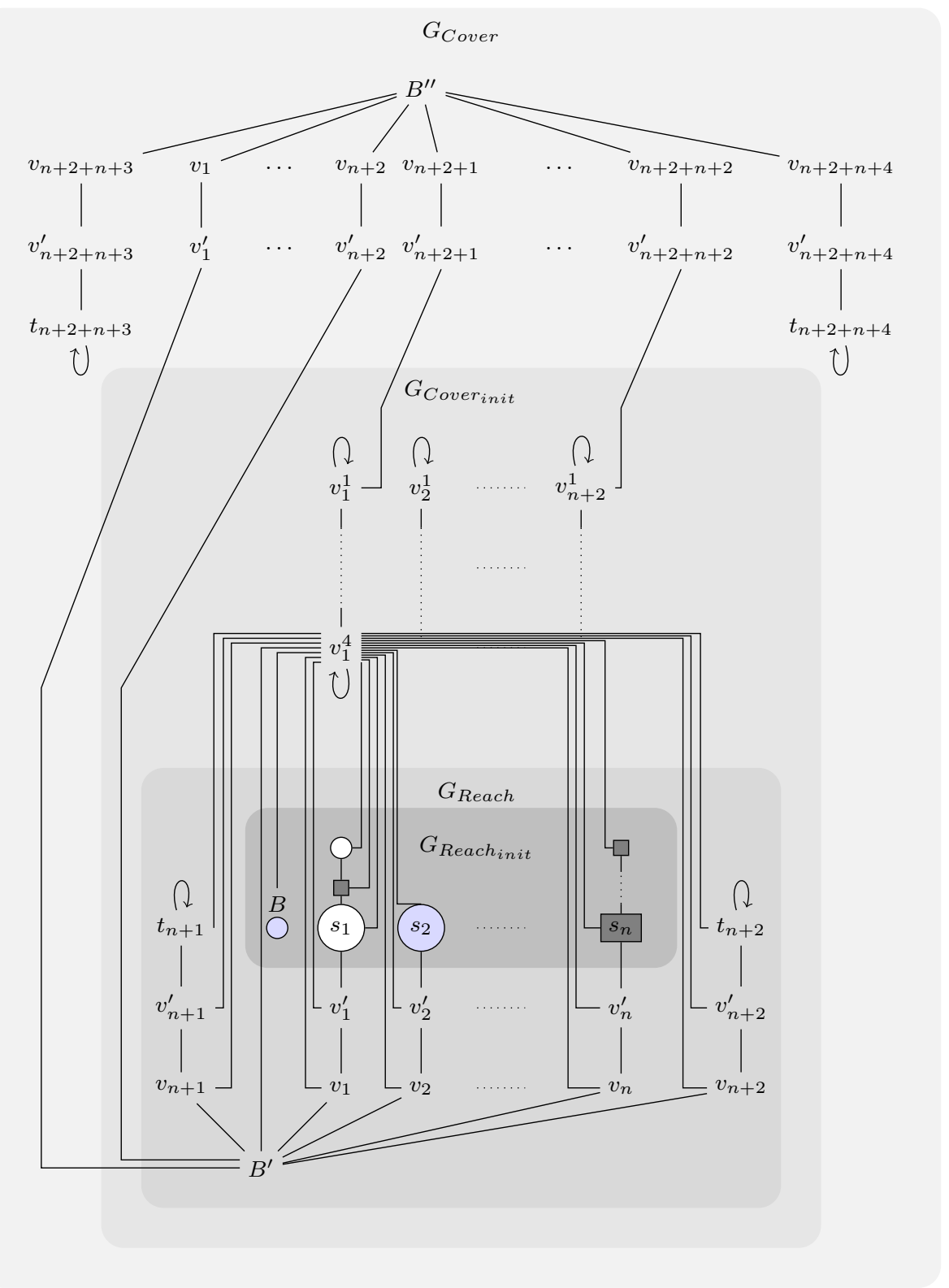

Fig. 13: Summary of all the planar constructions. 
${\text { Theorem } 16 \text { Reachability }_{\text {und }} \text {, Coverage-init }}_{\text {und }}$ and Coverage $_{\text {und }}$ are PSPACE-hard on planar graphs.

\section{Conclusion}

The main result of this paper is the introduction of sight-moveable topological graphs. Indeed, being able to decide in NLOGSPACE whether the reachability or the coverage can be done is an important improvement over the previous results. However, this class does not yield an improvement for the optimization of the execution.

We have studied numerous variants of the defined problems in the hope to give a complete overview of this new extension of the MAPF problem. Unfortunately, we still lack results for some of these variants. Furthermore, it is also unclear whether the combination of these variants can yield higher complexity. Additionally, there are still many variants of MAPF that have not been extended to these problems.

In realistic situation, the topological graph may not be fully known in advance. Indeed, the discretization may not represent faithfully the area in which the agents evolve. Thus, we attend to extend this work to incomplete knowledge in which the agents only have a over-approximation of the actual topological graph. While the agents move around the area they can observe the graph and update their knowledge.

In addition, if we consider the variants with bounded disconnection, introduced in Subsection 8.3, with incomplete knowledge we fall in the setting of imperfect information. In this setting, an agent can fail to reconnect during the mission and the cooperation needs to consider the possible cause of this disconnection to finish the plan.

Finally, not only can the area be partially known by the agents but some mishaps can happen during the mission. Indeed, an external actor might disable an agent and, in this setting, the group of agent should have to find the agent or finish the mission without it. In the weighted graph extension (Subsection 8.2), mishaps can also be due to strong wind which modifies the cost of the movement.

\section{Acknowledgements}

This work was partially supported by UAV Retina Funded by EIT Digital. Special thanks to François Bodin for initiating the idea of this work. We thank Eva Soulier for the provided work during her internship. We also thank Sophie Pinchinat for useful comments.

\section{References}

1. A. Ahmadzadeh, J. Keller, G. Pappas, A. Jadbabaie, and V. Kumar. An optimization-based approach to time-critical cooperative surveillance and coverage with UAVs. In O. Khatib, V. Kumar, and D. Rus, editors, Experimental Robotics: The 10th International Symposium on Experimental Robotics, pages 491-500, Berlin, Heidelberg, 2008. Springer Berlin Heidelberg.

2. F. Amigoni, J. Banfi, and N. Basilico. Multirobot exploration of communication-restricted environments: A survey. IEEE Intelligent Systems, 32(6):48-57, November 2017. 
3. S. P. Anbuudayasankar, K. Ganesh, and S. Mohapatra. Models for practical routing problems in logistics. Springer, 2016

4. F. Aurenhammer. Voronoi diagrams - A survey of a fundamental geometric data structure. ACM Comput. Surv., 23(3):345-405, 1991.

5. J. Banfi, N. Basilico, and F. Amigoni. Intractability of time-optimal multirobot path planning on 2D grid graphs with holes. IEEE Robotics and Automation Letters, 2(4):1941-1947, 2017.

6. J. Banfi, A. Q. Li, N. Basilico, I. Rekleitis, and F. Amigoni. Asynchronous multirobot exploration under recurrent connectivity constraints. In 2016 IEEE International Conference on Robotics and Automation (ICRA), pages 5491-5498, May 2016.

7. F. Bodin, T. Charrier, A. Queffelec, and F. Schwarzentruber. Generating plans for cooperative connected uavs. In Proceedings of the Twenty-Seventh International Joint Conference on Artificial Intelligence, IJCAI 2018, July 13-19, 2018, Stockholm, Sweden, pages 5811-5813, 2018.

8. T. Bylander. The computational complexity of propositional STRIPS planning. Artif. Intell., 69(12): $165-204,1994$.

9. T. M. Cabreira, L. B. Brisolara, and P. R. Ferreira Jr. Survey on coverage path planning with unmanned aerial vehicles. Drones, 3(1), 2019

10. K. Cesare, R. Skeele, Soo-Hyun Yoo, Yawei Zhang, and G. Hollinger. Multi-UAV exploration with limited communication and battery. In 2015 IEEE International Conference on Robotics and Automation (ICRA), pages 2230-2235, May 2015.

11. T. Charrier, A. Queffelec, O. Sankur, and F. Schwarzentruber. Reachability and coverage planning for connected agents. In Proceedings of AAMAS, Montreal, QC, Canada, May 13-17, 2019, pages 1874-1876, 2019

12. T. Charrier, A. Queffelec, O. Sankur, and F. Schwarzentruber. Reachability and coverage planning for connected agents. In Proceedings of the Twenty-Eighth International Joint Conference on Artificial Intelligence, IJCAI 2019, Macao, China, August 10-16, 2019, pages 144-150, 2019.

13. Y. Chen, H. Zhang, and M. Xu. The coverage problem in uav network: A survey. In Fifth International Conference on Computing, Communications and Networking Technologies (ICCCNT), July 2014.

14. H. Choset. Coverage for robotics - A survey of recent results. Ann. Math. Artif. Intell., 31(1-4):113$126,2001$.

15. H. Choset and P. Pignon. Coverage path planning: The boustrophedon cellular decomposition. In A. Zelinsky, editor, Field and Service Robotics, pages 203-209, London, 1998. Springer London.

16. S. A. Cook. A taxonomy of problems with fast parallel algorithms. Information and Control, 64(13):2-21, 1985.

17. T. Danner and L. E. Kavraki. Randomized planning for short inspection paths. In Proceedings 2000 ICRA. Millennium Conference. IEEE International Conference on Robotics and Automation. Symposia Proceedings (Cat. No.00CH37065), volume 2, pages 971-976 vol.2, April 2000.

18. B. Englot and F. Hover. Sampling-based coverage path planning for inspection of complex structures In International Conference on Automated Planning and Scheduling, 2012.

19. B. Englot and F. Hover. Planning complex inspection tasks using redundant roadmaps. In H. I. Christensen and O. Khatib, editors, Robotics Research: The 15th International Symposium ISRR, pages 327-343, Cham, 2017. Springer International Publishing.

20. R. A. Finkel and J. L. Bentley. Quad trees: A data structure for retrieval on composite keys. Acta Inf., $4: 1-9,1974$

21. G. Francès, M. Ramírez, N. Lipovetzky, and H. Geffner. Purely declarative action descriptions are overrated: Classical planning with simulators. In Twenty-Sixth International Joint Conference on Artificial Intelligence, 2017.

22. M. Fu, A. Kuntz, O. Salzman, and R. Alterovitz. Toward asymptotically-optimal inspection planning via efficient near-optimal graph search. In Proceedings of Robotics: Science and Systems, FreiburgimBreisgau, Germany, June 2019.

23. E. Galceran and M. Carreras. A survey on coverage path planning for robotics. Robotics and Autonomous Systems, 61(12):1258 - 1276, 2013.

24. N. Hazon and G. A. Kaminka. Redundancy, efficiency and robustness in multi-robot coverage. In Proceedings of the 2005 IEEE International Conference on Robotics and Automation, pages 735-741, April 2005.

25. R. A. Hearn and E. D. Demaine. Pspace-completeness of sliding-block puzzles and other problems through the nondeterministic constraint logic model of computation. Theoretical Computer Science, 343(1):72 - 96, 2005. Game Theory Meets Theoretical Computer Science.

26. G. A. Hollinger and S. Singh. Multirobot coordination with periodic connectivity: Theory and experiments. IEEE Trans. Robotics, 28(4):967-973, 2012. 
27. A. Itai, C. H. Papadimitriou, and J. L. Szwarcfiter. Hamilton paths in grid graphs. SIAM J. Comput., 11(4):676-686, 1982.

28. R. M. Karp. Reducibility among combinatorial problems. In Proceedings of a symposium on the Complexity of Computer Computations, 1972.

29. L. E. Kavraki, M. N. Kolountzakis, and J. . Latombe. Analysis of probabilistic roadmaps for path planning. In Proceedings of IEEE International Conference on Robotics and Automation, volume 4, pages 3020-3025 vol.4, April 1996.

30. P. N. Klein, S. Mozes, and O. Weimann. Shortest paths in directed planar graphs with negative lengths: A linear-space o $(n \log 2 \mathrm{n})$-time algorithm. ACM Trans. Algorithms, 6(2):30:1-30:18, Apr. 2010.

31. A. Knoll. A survey of octree volume rendering methods. In GI, the Gesellschaft für Informatik, page 87,2006

32. J. J. Kuffner and S. M. LaValle. Rrt-connect: An efficient approach to single-query path planning. In Proceedings 2000 ICRA. Millennium Conference. IEEE International Conference on Robotics and Automation. Symposia Proceedings (Cat. No.00CH37065), volume 2, pages 995-1001 vol.2, April 2000.

33. T. Kusnur, S. Mukherjee, D. M. Saxena, T. Fukami, T. Koyama, O. Salzman, and M. Likhachev. A planning framework for persistent, multi-UAV coverage with global deconfliction. CoRR, abs/1908.09236, 2019.

34. S. M. LaValle. Planning Algorithms. Cambridge University Press, 2006.

35. H. Ma, C. A. Tovey, G. Sharon, T. K. S. Kumar, and S. Koenig. Multi-agent path finding with payload transfers and the package-exchange robot-routing problem. In Proceedings of the Thirtieth AAAI Conference on Artificial Intelligence, February 12-17, 2016, Phoenix, Arizona, USA, pages 31663173, 2016.

36. R. Morris, C. S. Pasareanu, K. S. Luckow, W. Malik, H. Ma, T. K. S. Kumar, and S. Koenig. Planning, scheduling and monitoring for airport surface operations. In Planning for Hybrid Systems, Papers from the 2016 AAAI Workshop, Phoenix, Arizona, USA, February 13, 2016, 2016.

37. T. Nestmeyer, P. Robuffo Giordano, H. H. Bülthoff, and A. Franchi. Decentralized simultaneous multi-target exploration using a connected network of multiple robots. Auton. Robots, 41(4):989-1011, Apr. 2017.

38. M. Otte and N. Correll. Any-com multi-robot path-planning with dynamic teams: Multi-robot coordination under communication constraints. In O. Khatib, V. Kumar, and G. Sukhatme, editors, $E x-$ perimental Robotics: The 12th International Symposium on Experimental Robotics, pages 743-757, Berlin, Heidelberg, 2014. Springer Berlin Heidelberg.

39. R. Pandey, A. K. Singh, and K. M. Krishna. Multi-robot exploration with communication requirement to a moving base station. In 2012 IEEE International Conference on Automation Science and Engineering (CASE), pages 823-828, Aug 2012.

40. H. X. Pham, H. M. La, D. Feil-Seifer, and M. C. Deans. A distributed control framework of multiple unmanned aerial vehicles for dynamic wildfire tracking. IEEE Transactions on Systems, Man, and Cybernetics: Systems, pages 1-12, 2018.

41. D. Ratner and M. K. Warmuth. Finding a shortest solution for the $\mathrm{N} \times \mathrm{N}$ extension of the 15-puzzle is intractable. In Proceedings of the 5th National Conference on Artificial Intelligence. Philadelphia, PA, USA, August 11-15, 1986. Volume 1: Science, pages 168-172, 1986.

42. O. Reingold. Undirected connectivity in log-space. J. ACM, 55(4):17:1-17:24, 2008

43. I. Rekleitis, A. P. New, E. S. Rankin, and H. Choset. Efficient boustrophedon multi-robot coverage: an algorithmic approach. Annals of Mathematics and Artificial Intelligence, 52(2):109-142, Apr 2008.

44. M. N. Rooker and A. Birk. Multi-robot exploration under the constraints of wireless networking. Control Engineering Practice, 15(4):435 - 445, 2007.

45. M. R. K. Ryan. Exploiting subgraph structure in multirobot path planning. in Journal of Artificial Intelligence Research, 31:497-542, 2008.

46. W. J. Savitch. Relationships between nondeterministic and deterministic tape complexities. J. Comput. Syst. Sci., 1970.

47. J. T. Schwartz and M. Sharir. On the piano movers' problem: Iii. coordinating the motion of several independent bodies: The special case of circular bodies moving amidst polygonal barriers. The International Journal of Robotics Research, 2(3):46-75, 1983.

48. G. Sharon, R. Stern, A. Felner, and N. Sturtevant. Conflict-based search for optimal multi-agent path finding. In Proceedings of the Twenty-Sixth AAAI Conference on Artificial Intelligence, AAAI'12, pages 563-569. AAAI Press, 2012.

49. D. Silver. Cooperative pathfinding. In Proceedings of the First AAAI Conference on Artificial Intelligence and Interactive Digital Entertainment, AIIDE’05, page 117-122. AAAI Press, 2005. 
50. M. Sipser. Introduction to the theory of computation. PWS Publishing Company, 1997.

51. K. Solovey and D. Halperin. On the hardness of unlabeled multi-robot motion planning. The International Journal of Robotics Research, 35(14):1750-1759, 2016.

52. T. Standley. Finding optimal solutions to cooperative pathfinding problems. In Proceedings of the Twenty-Fourth AAAI Conference on Artificial Intelligence, AAAI'10, page 173-178. AAAI Press, 2010 .

53. N. Sturtevant. Benchmarks for grid-based pathfinding. Transactions on Computational Intelligence and AI in Games, 4(2):144 - 148, 2012.

54. D. Tateo, J. Banfi, A. Riva, F. Amigoni, and A. Bonarini. Multiagent connected path planning: Pspacecompleteness and how to deal with it. In Thirty-Second AAAI Conference on Artificial Intelligence, 2018

55. W. L. Teacy, J. Nie, S. McClean, and G. Parr. Maintaining connectivity in UAV swarm sensing. In 2010 IEEE Globecom Workshops, pages 1771-1776. IEEE, 2010.

56. H. Turner. Polynomial-length planning spans the polynomial hierarchy. In Logics in Artificial Intelligence, European Conference, JELIA 2002, Cosenza, Italy, September, 23-26, Proceedings, 2002.

57. M. Veloso, J. Biswas, B. Coltin, and S. Rosenthal. Cobots: Robust symbiotic autonomous mobile service robots. In Proceedings of the 24th International Conference on Artificial Intelligence, IJCAI'15, page 4423-4429. AAAI Press, 2015.

58. P. R. Wurman, R. D'Andrea, and M. Mountz. Coordinating hundreds of cooperative, autonomous vehicles in warehouses. In Proceedings of the 19th National Conference on Innovative Applications of Artificial Intelligence - Volume 2, IAAI'07, pages 1752-1759. AAAI Press, 2007.

59. Xiaoming Zheng, Sonal Jain, S. Koenig, and D. Kempe. Multi-robot forest coverage. In 2005 IEEE/RSJ International Conference on Intelligent Robots and Systems, pages 3852-3857, Aug 2005.

60. L. Xu. Graph Planning for Environmental Coverage. PhD thesis, Carnegie Mellon University, Pittsburgh, PA, August 2011

61. E. Yanmaz. Connectivity versus area coverage in unmanned aerial vehicle networks. In Proceedings of IEEE International Conference on Communications, ICC 2012, 2012.

62. J. Yu. Intractability of optimal multirobot path planning on planar graphs. IEEE Robotics and Automation Letters, 1(1):33-40, 2016

63. J. Yu and S. LaValle. Planning optimal paths for multiple robots on graphs. Proceedings - IEEE International Conference on Robotics and Automation, 042012.

64. J. Yu and S. M. LaValle. Multi-agent path planning and network flow. In E. Frazzoli, T. LozanoPerez, N. Roy, and D. Rus, editors, Algorithmic Foundations of Robotics X, pages 157-173, Berlin, Heidelberg, 2013. Springer Berlin Heidelberg.

65. J. Yu and D. Rus. Pebble motion on graphs with rotations: Efficient feasibility tests and planning algorithms. In H. L. Akin, N. M. Amato, V. Isler, and A. F. van der Stappen, editors, Algorithmic Foundations of Robotics XI - Selected Contributions of the Eleventh International Workshop on the Algorithmic Foundations of Robotics, WAFR 2014, 3-5 August 2014, Boğaziçi University, İstanbul, Turkey, volume 107 of Springer Tracts in Advanced Robotics, pages 729-746. Springer, 2014. 